\title{
ON THE CHERN CHARACTER IN HIGHER TWISTED K-THEORY AND SPHERICAL T-DUALITY
}

\author{
LACHLAN MACDONALD, VARGHESE MATHAI, AND HEMANTH SARATCHANDRAN
}

\begin{abstract}
In this paper, we construct for higher twists that arise from cohomotopy classes, the Chern character in higher twisted K-theory, that maps into higher twisted cohomology. We show that it gives rise to an isomorphism between higher twisted K-theory and higher twisted cohomology over the reals. Finally we compute spherical T-duality in higher twisted K-theory and higher twisted cohomology in very general cases.
\end{abstract}

\section{CONTENTS}

Introduction

1. Preliminaries 3

2. Higher Twisted K-theory and the Chern Character 5

2.1. The universal Fred $_{\mathscr{O}_{\infty} \otimes \mathscr{K} \text {-bundle over } S^{2 k+1}} 5$

2.2. The Chern character for the universal Fred $_{\mathscr{O}_{\infty} \otimes \mathscr{K} \text {-bundle over } S^{2 k+1}}$

2.3. The Chern character for smooth manifolds with a cohomotopy twist 10

2.4. The odd case 11

3. Spherical T-duality 14

3.1. Historical introduction 14

3.2. Spherical T-duality computations 15

3.3. Spherical T-dual pairs 15

3.4. Gysin sequence and the cohomology of $Z$ and $\widehat{Z} \quad 16$

3.5. Isomorphism of higher twisted cohomologies 17

3.6. K-theory of $Z$ and $\widehat{Z}$ when the base $M$ is torsion free 18

3.7. Isomorphism of higher Twisted $K$-theories when the base $M$ is torsion free 20

4. Spectral sequences 26

4.1. The higher twisted Atiyah-Hirzebruch spectral sequence 26

4.2. Singular de Rham theory 29

4.3. The higher twisted cohomology spectral sequence 33

4.4. Comparison of the spectral sequences 35

$\begin{array}{ll}\text { References } & 37\end{array}$

2010 Mathematics Subject Classification. Primary 81T30 Secondary 19L50, 58B15, 19 L10.

Key words and phrases. D-branes, higher twisted K-theory, higher twisted cohomology, Chern character, spherical T-duality, Cuntz algebras, cohomotopy. 


\section{INTRODUCTION}

One of the goals in this paper is to give an explicit formula for the Chern character in higher twisted K-theory. Such a formula may be used in string theory to compute the charge of a D-brane $x \in K_{H}^{0}(M)$ for a spacetime $M$ in the presence of a flux $H$, according to the equation

$$
\operatorname{charge}(x)=\sqrt{\widehat{A}(M)} \cup C h_{H}(x) \in H_{H}^{\text {even }}(M),
$$

cf. [40]. While it is well known to experts that the Chern character abstractly exists, as may be seen using methods of rational homotopy theory cf. [5, 22, 47], it turns out to be rather tricky to give an explicit formula for the Chern character in the general case. In the end we show that for every odd degree cohomotopy class of a manifold $M$ (which defines an odd degree cohomology class $H$ of $M$ ) we can define an $H$-twisted K-theory and produce a formula for the Chern character in this case. As explained below, higher twisted K-theory and higher twisted cohomology arise in String/M-theory, and the Chern character constructed relates these. We begin with a brief historical introduction.

$D$-branes, studied in $[42,43]$ are extended objects upon which open strings can end with Dirichlet boundary conditions and are fundamental to string theory. It was argued in $[40,52]$ that charges of $D$-branes take values in $K$-theory. In [9], twisted K-theory was proposed as a classification for D-branes in a background flux. The proposal was consolidated in [10, 38], where geometric representatives for twisted K-theory were defined, and so was the twisted Chern character. Later in [4] an alternate description of twisted K-theory was presented, and an alternate construction of the twisted Chern character presented in [5].

For an account of T-duality in the absence of a background flux, using K-theory, see [27]. In $[11,12]$, T-duality in the presence of a background flux was studied for spacetimes compactified on a principal circle bundle $Z$. It was established that there is a change in topology whenever either the principal circle bundle is nontrivial or the background flux is topologically nontrivial. It was also first established that T-duality gives an isomorphism of twisted K-theory groups,

$$
T: K_{H}^{*}(Z) \rightarrow K_{\hat{H}}^{*+1}(\hat{Z}),
$$

where $\hat{Z}$ is the T-dual principal circle bundle. In $[17,37,51]$ there are alternate approaches to proving this; via a T-duality classifying space and via noncommutative geometry.

In [13] the authors first used higher twisted K-theory and higher twisted cohomology in String Theory. The authors argue that the 7-twisted K-theory/cohomology classify certain conserved charges in type IIB supergravity. They then conclude that spherical T-duality, which is duality of principal $S U$ (2)-bundles with degree 7 flux, provides a one to one map between conserved charges in certain topologically distinct compactifications and also a novel electromagnetic duality on the fluxes. They also suggest that spherical T-duality preserves the spectra of certain spherical 3-branes that wrap $S^{3}$ cycles in some spacetime $X$, i.e. by replacing closed strings, described by $\operatorname{Maps}\left(S^{1}, X\right)$, by spherical 3-branes described by $\operatorname{Maps}\left(S^{3}, X\right)$. Spherical T-duality in case of nonprincipal $S U(2)$-bundles is studied in [14]. There are several models for higher twisted K-theory, cf. [23, 36, 45, 47]. The model of higher twisted K-theory in [41] using the topological K-theory of certain operator algebra bundles will be used here.

Here we consider oriented $S^{2 k-1}$-bundles $Z$ over $2 k$ dimensional closed oriented manifolds $M$. Let $H$ be a top degree twist on $Z, H \in H^{4 k-1}(Z, \mathbb{Z}) \cong \mathbb{Z}$. Assuming that $M$ is a torsion-free manifold, then we can compute the cohomology and K-theory of $Z$ using the Gysin sequence. We can also 
compute the $H$-twisted K-theory and $H$-twisted cohomology of $Z$. Suppose that the Euler class of $Z$ is $e \in H^{2 k}(M, \mathbb{Z}) \cong \mathbb{Z}$. Note that the pushforward map $\pi_{*}: H^{4 k-1}(Z, \mathbb{Z}) \rightarrow H^{2 k}(M, \mathbb{Z})$ is an isomorphism, where $\pi: Z \rightarrow M$ is the projection map. Define a spherical T-dual of $(Z, H)$ to be a

pair $(\hat{Z}, \hat{H})$ where the Euler class of $\hat{Z}$ is equal to $\pi_{*}(H)$. Let $\hat{\pi}: \hat{Z} \rightarrow M$ be the projection where again the pushforward map $\hat{\pi}_{*}: H^{4 k-1}(\hat{Z}, \mathbb{Z}) \rightarrow H^{2 k}(M, \mathbb{Z})$ is an isomorphism. Define $\hat{\pi}_{*}(\hat{H})$ to be equal to the Euler class $e$. Then we show that there are degree shifting isomorphisms $K_{H}^{\bullet}(Z) \cong$ $K_{\hat{H}}^{\bullet+1}(\hat{Z})$ and $H_{H}^{\bullet}(Z, \mathbb{Z}) \cong H_{\hat{H}}^{\bullet+1}(\hat{Z}, \mathbb{Z})$. Furthermore, in the case that $M$ is not torsion free, our Chern character formula allows one to obtain a rational degree shifting isomorphism $K_{H}^{\bullet}(Z) \otimes \mathbb{Q} \cong$ $K_{\hat{H}}^{\bullet+1}(\hat{Z}) \otimes \mathbb{Q}$ of the higher twisted K-theories.

In [33], they instead consider iterated higher algebraic K-theory and its higher twists. They also define an abstract Chern character which takes values in the cohomological counterpart of twisted iterated higher algebraic K-theory, and prove an abstract T-duality for certain spherical bundles with flux. It is hard to relate those results to the results of our paper except to say that they have a similar flavour.

Higher twisted K-theory arises because K-theory admits natural automorphisms given by tensoring with virtual line-bundles, that is virtual vector bundles of rank 1 . The main construction is that of the Chern character in higher twisted K-theory for twists arising from cohomotopy classes. We generalise and clarify some constructions in [5]. The relevant Atiyah-Hirzebruch spectral sequence in higher twisted K-theory is constructed, and, after taking a detour through singular de Rham theory to establish some essential facts, we also construct the relevant spectral sequence for higher twisted cohomology. We conclude that for for any higher twist arising from the cohomotopy set of the space, the Chern character can be constructed and is a real isomorphism between higher twisted K-theory and higher twisted cohomology. The Atiyah-Hirzebruch spectral sequence we give is instrumental to the spherical T-duality computations.

\section{Acknowledgements}

HS and VM were partially supported by funding from the Australian Research Council, through the Australian Laureate Fellowship FL170100020. LM and VM were partially supported by funding from the Australian Research Council, through the Discovery Project grant DP200100729. HS wishes to acknowledge Jarah Evslin for useful discussions about spectral sequences and their differentials.

All three authors would like to thank the two anonymous referees for their comments on the paper, which have helped to improve the exposition.

\section{PRELIMinARies}

To make the paper more self contained, we add some preliminaries on operator algebras that are used in the paper. The main references to this section are the books $[6,50]$ and the first two sections of the thesis [16].

We begin with the definition of the Cuntz algebra $\mathscr{O}_{\infty}$. It is the universal $C^{*}$-algebra generated by infinitly many generators $\left\{S_{1}, S_{2}, \ldots\right\}$ subject to the relations $S_{i}^{*} S_{i}=1$ for all $i$, and $\sum_{j=1}^{N} S_{j} S_{j}^{*} \leq 1$ for all $N \in \mathbb{N}$. It is a simple $C^{*}$-algebra with many important algebraic properties that have been thoroughly studied in the literature. 
We consider the short exact sequence of $C^{*}$-algebras,

$$
0 \rightarrow \mathscr{O}_{\infty} \otimes \mathscr{K} \rightarrow \mathscr{M}\left(\mathscr{O}_{\infty} \otimes \mathscr{K}\right) \stackrel{\pi}{\rightarrow} \mathscr{M}\left(\mathscr{O}_{\infty} \otimes \mathscr{K}\right) / \mathscr{O}_{\infty} \otimes \mathscr{K} \rightarrow 0
$$

where $\mathscr{K}$ denotes the algebra of compact operators on an infinite dimensional Hilbert space, $\mathscr{M}$ denotes the multiplier algebra. An operator $F \in \mathscr{M}\left(\mathscr{O}_{\infty} \otimes \mathscr{K}\right)$ is said to be $\mathscr{O}_{\infty} \otimes \mathscr{K}$-Fredholm if $\pi(F) \in G L\left(\mathscr{M}\left(\mathscr{O}_{\infty} \otimes \mathscr{K}\right) / \mathscr{O}_{\infty} \otimes \mathscr{K}\right)$, the group of invertible elements in $\mathscr{M}\left(\mathscr{O}_{\infty} \otimes \mathscr{K}\right) / \mathscr{O}_{\infty} \otimes \mathscr{K}$. Then the space of all $\mathscr{O}_{\infty} \otimes \mathscr{K}$-Fredholm operators

$$
\text { Fred }_{\mathscr{O}_{\infty} \otimes \mathscr{K}}=\pi^{-1}\left(G L\left(\mathscr{M}\left(\mathscr{O}_{\infty} \otimes \mathscr{K}\right) / \mathscr{O}_{\infty} \otimes \mathscr{K}\right)\right)
$$

is an open subset of the $C^{*}$-algebra $\mathscr{M}\left(\mathscr{O}_{\infty} \otimes \mathscr{K}\right)$, so it is a smooth Banach manifold.

From the short exact sequence above, one can deduce that

$$
K_{0}\left(\mathscr{O}_{\infty} \otimes \mathscr{K}\right) \cong \pi_{0}\left(\text { Fred }_{\mathscr{O}_{\infty} \otimes \mathscr{K}}\right) \cong \mathbb{Z}
$$

since Cuntz [20] has shown that $\mathscr{O}_{\infty} \otimes \mathscr{K}$ is KK-equivalent to $\mathbb{C}$. In fact, we deduce that

$$
\pi_{j}\left(\text { Fred }_{\mathscr{O}_{\infty} \otimes \mathscr{K}}\right) \cong \mathbb{Z} \text { if } j \text { is even, } \cong 0 \text { if } j \text { is odd }
$$

Pennig's model [41] of the higher twisted K-theory of a space $X$ uses principal Aut $\left(\mathscr{O}_{\infty} \otimes \mathscr{K}\right)$ bundles over $X$, which are classified by maps $\lambda: X \rightarrow B A u t\left(\mathscr{O}_{\infty} \otimes \mathscr{K}\right)$. By the associated bundle construction, any such principal bundle $P_{\lambda}$ is associated to an algebra bundle $\mathscr{A}_{\lambda}$ over $X$ with fibre $\mathscr{O}_{\infty} \otimes \mathscr{K}$. Then the higher twisted $\mathrm{K}$-theory of $X$ is by definition

$$
K_{\lambda}^{j}(X)=K_{j}\left(C_{0}\left(X, \mathscr{A}_{\lambda}\right)\right)
$$

where $C_{0}\left(X, \mathscr{A}_{\lambda}\right)$ denotes the $C^{*}$-algebra of sections of $\mathscr{A}_{\lambda}$ that vanish at infinity. The associated bundle construction may also be used to yield bundles $\Omega^{j}$ Fred $d_{\lambda}$ over $X$ with typical fibre $\Omega^{j}$ Fred $_{\mathscr{O}_{\infty} \otimes \mathscr{K}}$ (here $\Omega^{j}$ denotes the iterated based loop space, while $\operatorname{Aut}\left(\mathscr{O}_{\infty} \otimes \mathscr{K}\right)$ acts on

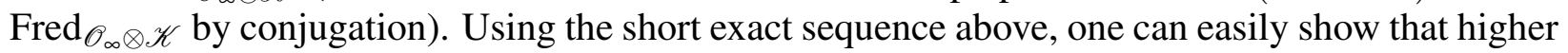
twisted K-theory can be equivalently described as

$$
K_{\lambda}^{j}(X)=\pi_{0}\left(C_{1}\left(X, \Omega^{j} \text { Fred }_{\lambda}\right)\right)
$$

where $C_{1}\left(M, \Omega^{j}\right.$ Fred $\left._{\lambda}\right)$ denotes the sections of the bundle $\Omega^{j}$ Fred $_{\lambda}$ that are equal to the identity outside of a compact set. If $A$ is a closed subset of $X$, then the higher twisted relative $\mathrm{K}$-theory is defined by

$$
K_{\lambda}^{j}(X, A):=K_{\lambda}^{j}(X \backslash A)
$$

There is a long exact sequence associated to relative higher twisted K-theory, which will be discussed later.

An important property of higher twisted K-theory, is that of Bott periodicity,

$$
K_{\lambda}^{j}(X) \cong K_{\lambda}^{j+2}(X)
$$

This says that it is enough to study $K_{\lambda}^{0}(X)$ and $K_{\lambda}^{1}(X)$. We remark here that from here on, indices in $\mathbb{Z}_{2}$ will always be shown in bold font. Thus the $\mathbb{Z}_{2}$-graded $K$-theory groups will be denoted $K_{\lambda}^{\mathbf{0}}(X)$ and $K_{\lambda}^{\mathbf{1}}(X)$.

One final remark on higher twisted K-theory is that twists on a space $X$ in the general sense outlined above need not come from cohomology classes for $X$. This is to be contrasted to ordinary twisted Ktheory, in which twists always arise from integral cohomology classes. In the special cases where 
the twist does correspond to a cohomology class $[H] \in H^{*}(X ; \mathbb{Z})$, the higher twisted K-theory will be denoted

$$
K_{[H]}^{j}(X)
$$

instead of $K_{\lambda}^{j}(X)$. Examples of this include when $X$ is a finite, connected, torsion-free CW complex [21, Corollary 4.4] (and in particular whenever $X$ is a compact, torsion-free, connected manifold). We recall from the work of M. Dadarlat and U. Pennig in [21] that the homotopy groups for $\operatorname{BAut}\left(\mathscr{O}_{\infty} \otimes \mathscr{K}\right)$ were computed to be $\mathbb{Z}_{2}$ in degree $1, \mathbb{Z}$ in all other odd degrees and 0 otherwise. An important class of examples for us are those for which the twist $\lambda: X \rightarrow B A u t\left(\mathscr{O}_{\infty} \otimes \mathscr{K}\right)$ factors through an odd sphere, so that $\lambda=f \circ H$, where $f: S^{2 k+1} \rightarrow \operatorname{BAut}\left(\mathscr{O}_{\infty} \otimes \mathscr{K}\right)$ is the generator of the $(2 k+1)$ homotopy group, and where $H: X \rightarrow S^{2 k+1}$ defines a class in the cohomotopy set of $X$, hence a class $[H] \in H^{2 k+1}(X ; \mathbb{Z})$. Note that by the arguments of [4], such cohomotopy twists generalise the ordinary twists already familiar from string theory.

Higher twisted cohomology was studied in detail in [39], and will also be discussed later in the paper.

\section{Higher Twisted K-Theory And the Chern Character}

The goal of this section is to define a Chern character for higher twisted K-theory, when the twist is given by a cohomotopy class. We start by using work of M. Dadarlat and U. Pennig in [21] to

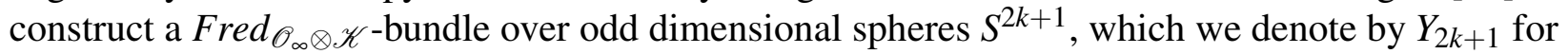
$k \geq 1$. Generalising the method of Atiyah and Segal in [4], we then show how to construct a Chern character for the bundle $Y_{2 k+1}$. This Chern character takes values in a higher twisted cohomology group, and has the property that it lifts the classical Chern character of Fred $_{\mathscr{O}_{\infty} \otimes \mathscr{K}}$. Finally, using the constructed Chern character for the bundle $Y_{2 k+1}$, we are able to construct a Chern character for a compact smooth manifold $X$, with a fixed cohomotopy class $[\lambda] \in\left[X, S^{2 k+1}\right]$ as twist. Analogous to the case of $Y_{2 k+1}$, the Chern character for $X$ lands in a twisted Cohomology group, determined by the cohomotopy twist $[\lambda]$. Our strategy can be seen as a generalisation of the methods employed by Atiyah and Segal in [4], where a Chern character for 3-twisted K-theory is constructed by first constructing one over a universal Fred-bundle over $S^{3}$.

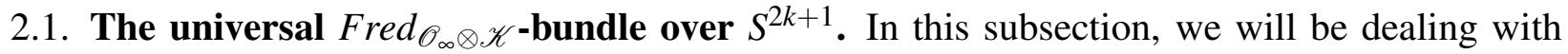
Fred $_{\mathscr{O}_{\infty} \otimes \mathscr{K}}$-bundles over compact smooth manifolds. Such bundles are classified by maps into the classifying space $B A u t\left(\right.$ Fred $\left._{\mathscr{O}_{\infty} \otimes \mathscr{K}}\right)$, and can be given as the pull-back of the universal Fred $_{\mathscr{O}_{\infty} \otimes \mathscr{K}^{-}}$ bundle over $B A u t\left(\right.$ Fred $\left._{\mathscr{O}_{\infty} \otimes \mathscr{K}}\right)$, which we will denote by Fred $(P)$. The reader is invited to consult section 1 for preliminaries about such bundles, and the references there in for a more comprehensive discussion.

The cohomology generator $u_{2 k+1} \in H^{2 k+1}\left(S^{2 k+1} ; \mathbb{Z}\right)$ gives rise to a Fred $\mathscr{O}_{\infty} \otimes \mathscr{K}$-bundle, denoted $Y_{2 k+1}$, via corollary 4.7 (ii) in [21], and the associated bundle construction, see section 1 . In fact, from the referenced corollary, we see that every cohomology class in $H^{2 k+1}\left(S^{2 k+1} ; \mathbb{Z}\right)$ gives rise to a Fred $\mathscr{O}_{\infty} \otimes \mathscr{K}$-bundle, and the one corresponding to the generator $u_{2 k+1}$ can be thought of as generating all these bundles. In this regard, we can think of $Y_{2 k+1}$ as a universal $F$ red $\mathscr{O}_{\infty} \otimes \mathscr{K}$-bundle over $S^{2 k+1}$. 
It will be important for us to understand the Serre spectral sequence associated to the bundle $Y_{2 k+1} \rightarrow S^{2 k+1}$. As the base of the Serre spectral sequence is $S^{2 k+1}$, we see that the only nontrivial differential is $d_{2 k+1}: E_{2 k+1}^{0, q} \rightarrow E_{2 k+1}^{2 k+1, q-2 k}$, and the spectral sequence reduces to the Wang exact sequence.

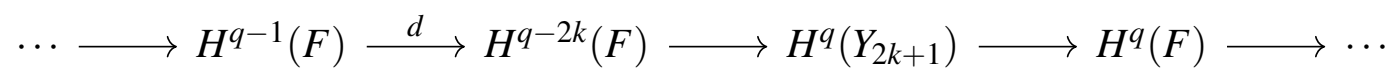

where $d=d_{2 k+1}$ is the differential of the Serre spectral sequence and $F=$ Fred $_{\mathscr{O}_{\infty} \otimes \mathscr{K}}$.

Any Fred $_{\mathscr{O}_{\infty} \otimes \mathscr{K}}$-bundle, over $S^{2 k+1}$, can be constructed by taking two trivial bundles over each $D^{2 k+1}$ hemisphere of $S^{2 k+1}$, and gluing them together via a gluing map. In other words, such a bundle is determined via its clutching map.

$$
\psi: S^{2 k} \times \text { Fred }_{\mathscr{O}_{\infty} \otimes \mathscr{K}} \rightarrow S^{2 k} \times \text { Fred }_{\mathscr{O}_{\infty} \otimes \mathscr{K}}
$$

The following proposition gives the action of $\psi^{*}$ on cohomology.

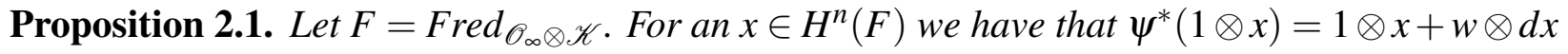

Proof. We have the following commutative diagram

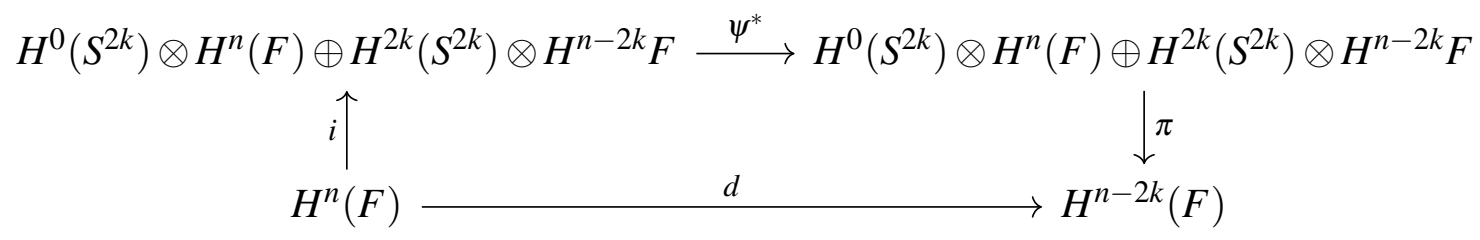

where we have used Kunneth's theorem to write $H^{n}\left(S^{2 k} \times F\right)=H^{0}\left(S^{2 k}\right) \otimes H^{n}(F) \oplus H^{2 k}\left(S^{2 k}\right) \otimes$ $H^{n-2 k} F, i(x)=1 \otimes x$ denotes the inclusion and $\pi$ the projection.

On taking the trivial clutching function, we see that the first term of $\psi^{*}(1 \otimes x)$ must be $1 \otimes x$. Together with the above commutative diagram, it follows that $\psi^{*}(1 \otimes x)=1 \otimes x+w \otimes d x$.

Given a compact space $X$ it is well known that the Picard group Pic $(X)$, formed by (equivalence classes of) line bundles on $X$, is a multiplicative subgroup of $K(X)$. Hence, there exists an action

$$
\operatorname{Pic}(X) \times K(X) \rightarrow K(X)
$$

by group homomorphisms. On the level of vector bundles, this action is induced by tensoring with a line bundle: $(L, E) \mapsto L \otimes E$.

Atiyah and Segal, see p. 5-7 in [4], were able to use this action to understand the differential $d_{3}$ in the Serre spectral sequence of a universal Fred-bundle over $S^{3}$. This provided a convenient strategy for them to define the Chern character for twisted $K$-theory twisted by a degree 3 cohomology class. We will be taking a similar approach, and as the bundles we are working with are slightly more general. We will need to understand a more general action.

The above action is a special case of a more general action given by virtual line bundles, which we now describe. Consider the rank function

$$
r k: K(X) \rightarrow \mathbb{Z}
$$

that gives the rank of a virtual vector bundle in $K(X)$. 
Let $\operatorname{VPic}(X):=r k^{-1}(1)$. An element of $\operatorname{VPic}(X)$ is a virtual line bundle. We point out that while $\operatorname{Pic}(X)$ is a multiplicative group, $V \operatorname{Pic}(X)$ is only a set. An element of $V \operatorname{Pic}(X)$ does not necessarily have a multiplicative inverse. We call VPic $(X)$ the virtual Picard set on $X$. These facts give us an action

$$
\operatorname{VPic}(X) \times K(X) \rightarrow K(X)
$$

of virtual line bundles on the $K$-theory of $X$, induced via tensor product.

The definition of the virtual Picard set looks very similar to that of the Picard group. However, there is a key difference. It is well know that there exists an isomorphism

$$
c_{1}: \operatorname{Pic}(X) \rightarrow H^{2}(X ; \mathbb{Z})
$$

induced via the first Chern class. This implies that $\operatorname{Pic}(X)$ is a cohomological action on $K(X)$. In the case of $V P i c(X)$, there is no such isomorphism. In other words, the action of $V P i c(X)$ on $K(X)$ is not cohomological in any way, and is an honest action coming from the $K$-theory of $X$ as opposed to the singular cohomology of $X$. This action will be used shortly to understand the differential $d_{2 k+1}$ in the Serre spectral sequence of the bundle $Y_{2 k+1}$.

Since for compact spaces $X, K^{0}(X) \cong\left[X\right.$, Fred $\left._{\mathscr{O}_{\infty} \otimes \mathscr{K}}\right] \cong[X$, Fred $]$, we can use Yoneda's lemma to conclude that Fred $_{\mathscr{O}_{\infty} \otimes \mathscr{K}}$ and Fred are homotopy equivalent. Therefore cohomology ring of Fred $_{\mathscr{O}_{\infty} \otimes \mathscr{K}}$ is given by the following proposition.

Proposition 2.2. We have an isomorphism of rings $H^{*}\left(\right.$ Fred $\left._{\mathscr{O}_{\infty} \otimes \mathscr{K}} ; \mathbb{Z}\right) \cong \mathbb{Z}\left[s_{1}, s_{2}, \ldots, s_{n}, \ldots\right]$, where $s_{i} \in H^{2 i}\left(\right.$ Fred $\left._{\mathscr{O}_{\infty} \otimes \mathscr{K}} ; \mathbb{Z}\right)$.

The element $s_{0} \in H^{0}\left(\right.$ Fred $\left._{\mathscr{O}_{\infty} \otimes \mathscr{K}} ; \mathbb{Z}\right)$ corresponds to the index. Letting Fred $_{\mathscr{O}_{\infty} \otimes \mathscr{K}}^{k}$ denote the component of Fred $_{\mathscr{O}_{\infty} \otimes \mathscr{K}}$ consisting of index $k$ operators, we have that $s_{0}=k \in H^{0}\left(\right.$ Fred $\left._{\mathscr{O}_{\infty} \otimes \mathscr{K}}^{k} ; \mathbb{Z}\right)$. In particular, in the index zero component $s_{0}=0$.

We will also need the following theorem of Bott, see corollary 9.8 in [28].

Theorem 2.3 (Bott). Let $u \in H^{2 n}\left(S^{2 n} ; \mathbb{Z}\right)$ be a generator. Given any rank $n$ complex vector bundle $E$ over $S^{2 n}$ we have $c_{n}(E)=m(n-1)$ ! u for some $m \in \mathbb{Z}$, where $c_{n}(E)$ denotes the $n$th Chern class of $E$.

We remark that $\widetilde{K}\left(S^{2 n}\right)$ can be generated by an element of the form $E-\underline{n}$, where $E$ is a rank $n$ complex vector bundle and $\underline{n}$ denotes a trivial rank $n$ bundle so the Chern character of $E-\underline{n}$ must be non-zero. So in the above theorem, we can always find a $E$ with $c_{n}(E) \neq 0$.

Bott's theorem will be applied to compute the differential $d_{2 k+1}$ in the Serre spectral sequence associated to the bundle $Y_{2 k+1} \rightarrow S^{2 k+1}$. Before we can show how this is done, we give the reader a reminder of the theory of Chern classes and the Chern character.

Given a rank $n$ vector bundle, we define the Chern classes via the formal variables $x_{1}, \ldots, x_{n}$ so that

$$
\sum_{i=0}^{n} c_{i}=\prod_{i=1}^{n}\left(1+x_{i}\right)
$$

and define the power sums $s_{n}$ by

$$
s_{n}=\sum_{i=1}^{n} x_{i}^{n} .
$$


The importance of the power sums comes from the fact that they give a nice definition for the Chern character of a vector bundle $E$

$$
C h(E)=\sum_{n=0}^{\infty} \frac{s_{n}}{n !} .
$$

Given two vector bundles $E$ and $F$ we have that $C h(E \otimes F)=C h(E) C h(F)$, and hence we obtain the formula

$$
s_{n}(E \otimes F)=\left(\begin{array}{l}
n \\
0
\end{array}\right) s_{n}(E) s_{0}(F)+\cdots+\left(\begin{array}{l}
n \\
k
\end{array}\right) s_{n-k}(E) s_{k}(F)+\cdots+s_{0}(E) s_{n}(F) .
$$

A particular special case of the above formula will be important in our context. Fix a rank $k$ complex vector bundle $E$ over a $2 k$-dimensional manifold $X$, such that $c_{m}(E)=0$ for all $1 \leq m \leq$ $k-1$ and $c_{k}(E)=v \in H^{2 k}(X ; \mathbb{Z})$. Then for any $F \in K(X)$, letting $s_{n}$ denote the power sums for $F$ and $s_{n}(u)$ the power sums for $E \otimes F$, we have

$$
s_{n}(u)=s_{n}+\frac{(-1)^{k+1} n !}{(k-1) !(n-k) !} v s_{n-k} .
$$

We can use this to obtain a formula for the differential $d_{2 k+1}$ in the Serre spectral sequence for the bundle $Y_{2 k+1}$ over $S^{2 k+1}$.

Proposition 2.4. In the Serre spectral sequence for the bundle $Y_{2 k+1} \rightarrow S^{2 k+1}$, with fibre Fred $d_{\mathscr{O}_{\infty} \otimes \mathscr{K}}^{j}$ and cohomology with $\mathbb{R}$-coefficients, the differential $d_{2 k+1}$ is given on the cohomology generators $s_{n}$ by

$$
\begin{aligned}
& d_{2 k+1} s_{n}=\frac{(-1)^{k+1} n !}{(n-k) !} m u_{2 k+1} s_{n-k} \text { for } n \neq k \\
& d_{2 k+1} s_{k}=(-1)^{k+1} k ! m u_{2 k+1} s_{0}=(-1)^{k+1} k ! m j u_{2 k+1}
\end{aligned}
$$

where $u_{2 k+1} \in H^{2 k+1}\left(S^{2 k+1} ; \mathbb{Z}\right)$ is a generator and $0 \neq m \in \mathbb{Z}$.

Proof. Let $u_{2 k} \in H^{2 k}\left(S^{2 k} ; \mathbb{Z}\right)$ be a generator that suspends to the generator $u_{2 k+1} \in H^{2 k+1}\left(S^{2 k+1} ; \mathbb{Z}\right)$. Applying Bott's theorem 2.3, we can find a rank $n$ bundle $E$ such that $c_{n}(E)=m(n-1) ! u$, for some integer $m \neq 0$. We then consider the virtual line bundle $\widetilde{E}:=E-\underline{n-1}$, where $\underline{n-1}$ denotes the trivial rank $n-1$ bundle on $S^{2 k}$.

By tensoring with $\mathbb{R}$, we let $\widetilde{E}_{\mathbb{R}}$ denote the 1 -dimensional $\mathbb{R}$-subspace of $\operatorname{VPic}\left(S^{2 k}\right) \otimes \mathbb{R}$ generated by $\widetilde{E}$. Then $c_{n}$ extends to an isomorphism

$$
c_{n}: \widetilde{E}_{\mathbb{R}} \rightarrow H^{2 k}\left(S^{2 k} ; \mathbb{R}\right) .
$$

The above map, together with the fact Fred $_{\mathscr{O}_{\infty} \otimes \mathscr{K}}$ represent a classifying space for $K$-theory, imply that cohomologically the action of the clutching map is given by formula 2.3 with $v=m(k-1) ! u$. Applying proposition 2.1, we obtain the formula for $d_{2 k+1}$.

Observe that the spectral sequence gives rise to the exact sequence

$$
H^{2 k}\left(\text { Fred }_{\mathscr{O}_{\infty} \otimes \mathscr{K}}^{j}\right) \stackrel{d_{2 k+1}}{\longrightarrow} H^{2 k+1}(X) \stackrel{\pi^{*}}{\longrightarrow} H^{2 k+1}\left(\text { Fred }_{\mathscr{O}_{\infty} \otimes \mathscr{K}}^{j}\right)
$$


where $\pi: Y_{2 k+1} \rightarrow S^{2 k+1}$ denotes the projection. We thus see that

$$
\begin{aligned}
\pi^{*} d_{2 k+1} s_{k} & =\pi^{*}\left((-1)^{k+1} k ! m j u_{2 k+1}\right) \\
& =(-1)^{k+1} k ! m j \pi^{*} u_{2 k+1} \\
& =0
\end{aligned}
$$

This implies $\pi^{*}\left(u_{2 k+1}\right)=0$ if $j \neq 0$. If the bundle $Y_{2 k+1}$ posses a section then $\pi^{*}$ is injective. As $u_{2 k+1} \neq 0 \in H^{2 k+1}\left(S^{2 k+1} ; \mathbb{Z}\right)$ it follows that $j=0$. Therefore we find that there are no sections with non-zero index. This means that when we define higher twisted K-theory for a smooth compact manifold $X$ twisted by a cohomotopy class, represented by a map $\lambda: X \rightarrow S^{2 k+1}$, we need only

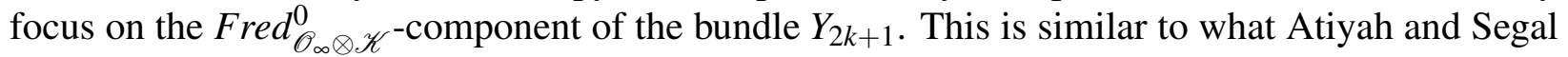
observe for 3-twisted $K$-theory, see p. 7 in [4].

2.2. The Chern character for the universal Fred $_{\mathscr{O}_{\infty} \otimes \mathscr{K}}$-bundle over $S^{2 k+1}$. In this subsection, we will define a Chern character for the bundle $Y_{2 k+1}$ that will land in a higher twisted cohomology group. This Chern character can be seen as a lift of the classical Chern character on Fred $\mathscr{O}_{\infty} \otimes \mathscr{K}$, and will play a pivotal role in the next subsection where we define a Chern character for smooth compact manifolds with a fixed cohomotopy twist.

We recall that $s_{n} \in H^{2 n}\left(\right.$ Fred $\left._{\mathscr{O}_{\infty} \otimes \mathscr{K}}\right)$ represents a cohomological generator, given by proposition 2.2.

We will let $\eta=\pi_{2 k+1}^{*} u_{2 k+1} \in H^{2 k+1}\left(Y_{2 k+1} ; \mathbb{R}\right)$, where $u_{2 k+1} \in H^{2 k+1}\left(S^{2 k+1} ; \mathbb{R}\right)$ is a generator represented by a closed form whose support does not contain the poles of $S^{2 k+1}$. Furthermore, let $F_{\infty}$ denote the fibre of $Y_{2 k+1}$ over the north pole of $S^{2 k+1}$, which we denote by $\{\infty\}$, and $F_{0}$ the fibre of $Y_{2 k+1}$ over the south pole, which we denote by $\{0\}$.

As mentioned at the end of section 2.1, there are no sections of non-zero index in higher twisted

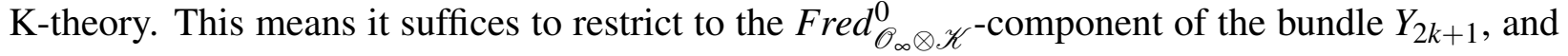
we shall do this from here on in.

In order to construct a Chern character, we will start by constructing forms $S_{n}$ on $Y_{2 k+1}$, for $n \geq 0$, that satisfy the following two conditions:

(1) $\left.S_{n}\right|_{F_{0}}=s_{n}$

(2) $d S_{n}=\frac{(-1)^{k+1} n !}{(n-k) !} m \eta S_{n-k}=\frac{(-1)^{k+1} n !}{(n-k) !} \eta_{2 k+1} S_{n-k}$

where we remind the reader that the coefficient of the right hand side of (2) comes from proposition 2.4, and we have defined $\eta_{2 k+1}=m \eta$.

The construction proceeds via induction on $n$. We define $S_{0}=0$. Suppose we have constructed $S_{m}$ for all $m<n$ that satisfies the above two conditions. Our goal is to construct $S_{n}$.

The bundle $Y_{2 k+1}$ can be trivialised over $S^{2 k+1} \backslash\{\infty\}$, we have that $Y_{2 k+1} \backslash F_{\infty} \cong D^{2 k+1} \times F_{r e d} \mathscr{O}_{\infty} \otimes \mathscr{K}$. In particular, we can pull back the form $s_{n}$ on Fred $_{\mathscr{O}_{\infty} \otimes \mathscr{K}}$, obtaining a form $s_{n}^{\prime}$ on $Y_{2 k+1} \backslash F_{\infty}$.

Let $\rho$ be a bump function on $Y_{2 k+1}$ supported away from $F_{\infty}$. Define $S_{n}^{\prime}:=\rho s_{n}^{\prime}$. By definition, $S_{n}^{\prime}$ is an $2 n$ form defined on all of $Y_{2 k+1}$. However, $S_{n}^{\prime}$ is not necessarily closed on all of $Y_{2 k+1}$. We do have that $d S_{n}^{\prime}=0$ outside a small neighbourhood of $F_{\infty}$ (i.e. in a small neighbourhood of $F_{0}$ ). As $d\left(d S_{n}^{\prime}\right)=0$ globally on $Y_{2 k+1}$, and $d S_{n}^{\prime}=0$ in a neighbourhood of $F_{0}$, we have that $d S_{n}^{\prime}$ defines a class in the relative group $H^{2 n+1}\left(Y_{2 k+1}, F_{0}\right)$. By proposition 2.4 this class can also be represented 
by $\frac{(-1)^{k+1} n !}{(n-k) !} m \eta S_{n-k}$. It follows that on the level of forms we have

$$
d S_{n}^{\prime}-\frac{(-1)^{k+1} n !}{(n-k) !} m \eta S_{n-k}=d \omega_{n}
$$

where $\omega_{n}$ is a $2 n$-form on $Y_{2 k+1}$ that is supported away from $F_{\infty}$. We then define $S_{n}:=S_{n}^{\prime}-\omega_{n}$. It is clear that $S_{n}$ satisfies conditions (1) and (2).

We can then define an even form $C h_{2 k+1}^{\mathbf{0}}$ on $Y_{2 k+1}$ by

$$
C h_{2 k+1}^{\mathbf{0}}=\sum_{n=1}^{\infty} \frac{S_{n}}{n !} .
$$

We observe that $D_{\eta_{2 k+1}} C h_{2 k+1}^{\mathbf{0}}=\left(d-\eta_{2 k+1}\right) C h_{2 k+1}^{\mathbf{0}}=0$, and hence $C h_{2 k+1}^{\mathbf{0}}$ defines a cocycle for higher twisted cohomology, with twist given by the cohomology class $\left[\eta_{2 k+1}\right]$, of the bundle $Y_{2 k+1}$. Therefore $C h_{2 k+1}^{\mathbf{0}} \in H_{\eta_{2 k+1}}^{\mathbf{0}}\left(Y_{2 k+1}\right)$, the even twisted cohomology group, and from condition (1) above we see that it lifts the classical Chern character in $H^{*}\left(\right.$ Fred $\left._{\mathscr{O}_{\infty} \otimes \mathscr{K}}\right)$.

2.3. The Chern character for smooth manifolds with a cohomotopy twist. In this subsection we use the Chern character from the previous subsection to construct a Chern character for smooth compact manifolds $X$. Our Chern character has the restriction that it can only be defined for twists corresponding to cohomotopy classes $\left[X, S^{2 k+1}\right]$. This latter set is known as the $2 k+1$ cohomotopy set of $X$. In general, it is just a set and not a group. However, we point out that if $X$ is a finite $\mathrm{CW}$-complex of dimension $n$, where $n \leq 2 k+1$, then the cohomology group $H^{2 k+1}(X ; \mathbb{Z})$ is given by the cohomotopy classes $\left[X, S^{2 k+1}\right]$. This follows from the fact that elements in $H^{2 k+1}(X ; \mathbb{Z})$ are represented by maps into the Eilenberg-Maclane space $K(\mathbb{Z}, 2 k+1)$, and by cellular approximation such a map can be assumed to be contained in the $2 k+1$-skeleton of $K(\mathbb{Z}, 2 k+1)$, which is $S^{2 k+1}$. Furthermore, if $X$ is a finite CW complex of dimension $n \leq 2 k-2$, then by work of Borsuk, see [7] and chapter 2 section 11 in [8], it is known that $\pi^{k}(X)$ is an abelian group. In our case, $X$ will always be a smooth compact manifold, hence can be given the structure of a finite CW complex. Thus if $\operatorname{dim}(X) \leq 2 k+1$, we have that $H^{2 k+1}(X ; \mathbb{Z})=\left[X, S^{2 k+1}\right]$ and hence any twist corresponding to a class in $\left[X, S^{2 k+1}\right]$ is necessary cohomological. However, if $\operatorname{dim}(X)>2 k+1$ then elements of $\left[X, S^{2 k+1}\right]$ could determine twists that are not necessarily cohomological. One final point to make is that our Chern character we define for $X$ will be defined via a pull back of the smooth form $C h_{2 k+1}^{\mathbf{0}}$. Therefore, we will need to restrict ourselves to smooth maps from $X$ to $S^{2 k+1}$. This will not cause any problems as each class in $\left[X, S^{2 k+1}\right]$ has a (not necessarily unique) smooth representative. When taking representatives of such cohomotopy classes, we will always be taking smooth ones without saying.

We fix a cohomotopy class $[\lambda] \in\left[X, S^{2 k+1}\right]$, represented by a smooth map $\lambda: X \rightarrow S^{2 k+1}$. We define

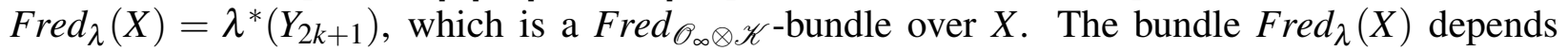
on the representative map $\lambda: X \rightarrow S^{2 k+1}$, however its isomorphism class only depends on the homotopy class of $\lambda$.

Following Atiyah and Segal's approach in [4], we define the higher twisted K-theory, twisted by the class $\lambda$, as follows 
Definition 2.5. Given a smooth compact manifold $X$, and a cohomotopy class $[\lambda] \in\left[X, S^{2 k+1}\right]$, represented by $\lambda: X \rightarrow S^{2 k+1}$, we define the higher twisted $K$-theory of $X$, twisted by the cohomotopy class $[\lambda]$ and denoted $K_{\lambda}^{0}(X)$, to consist of homotopy classes in $\left[X, Y_{2 k+1}\right]$ that lift the map $\lambda$. In other words, an element of $K_{\lambda}^{0}(X)$ is a homotopy class of a smooth map $F: X \rightarrow Y_{2 k+1}$ such that the following digram commutes

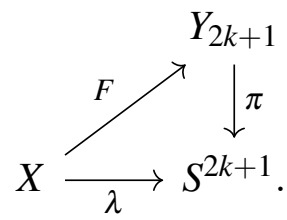

We remark that an equivalent way to define $K_{\lambda}^{\mathbf{0}}(X)$ would be as homotopy classes of sections of the bundle $\operatorname{Fred}_{\lambda}(X)$. This follows from the fact that $\operatorname{Fred}_{\lambda}(X)=\lambda^{*} Y_{2 k+1}$. We also remark that as Fred $_{\mathscr{O}_{\infty} \otimes \mathscr{K}}$ has the structure of a smooth Banach manifold, see section 1, it follows that $Y_{2 k+1}$ can be given the structure of a smooth Banach manifold. Therefore, the concept of a smooth map $F: X \rightarrow Y_{2 k+1}$ is well defined.

The group structure on $K_{\lambda}^{\mathbf{0}}(X)$ comes from the group structure of the fibres of the bundle $Y_{2 k+1}$. I.e. given $[F]$ and $[G] \in K_{\lambda}^{\mathbf{0}}(X)$, we define $[F]+[G]$ to be the class $[F+G]$. Note that $F+G$ is defined as the map such that $F+G(x):=F(x)+G(x)$, which is well defined because $F(x), G(x) \in \pi^{-1}(f(x))$ and hence $F(x)+G(x) \in \pi^{-1}(f(x))$. By Bott periodicity, we see that the groups $K_{\lambda}^{\mathbf{0}}(X)$ are the even degree groups of the twisted $K$-theory of $X$. The odd case will be defined in the next subsection.

We now define a twisted Chern character for $X$. We recall towards the end of the previous section we defined a Chern character $C h_{2 k+1}^{\mathbf{0}} \in H_{\eta_{2 k+1}}\left(Y_{2 k+1}\right)$. We also recall that the cohomology class $\eta_{2 k+1} \in H^{2 k+1}\left(Y_{2 k+1}\right)$ was chosen via pulling back a particular generator of $H^{2 k+1}\left(S^{2 k+1}\right)$, which we denoted $u_{2 k+1}$. Therefore, given a class $[F] \in K_{\lambda}^{\mathbf{0}}(X)$, represented by a smooth map $F: X \rightarrow$ $Y_{2 k+1}$, we have $F^{*}\left(\eta_{2 k+1}\right)=F^{*} \pi^{*}\left(u_{2 k+1}\right)=\lambda^{*}\left(u_{2 k+1}\right)$.

We define the twisted Chern character

$$
C h_{\lambda}^{\mathbf{0}}: K_{\lambda}^{\mathbf{0}}(X) \rightarrow H_{\lambda^{*}\left(u_{2 k+1}\right)}^{\mathbf{0}}(X)
$$

by $C h_{\lambda}^{\mathbf{0}}([F])=\left[F^{*} C h_{2 k+1}^{\mathbf{0}}\right]$, where $F$ is a smooth representative. The fact that this defines an element of the twisted cohomology group $H_{\lambda *\left(u_{2 k+1}\right)}(X)$ follows by naturality and the fact that $C h_{2 k+1}^{\mathbf{0}} \in H_{\eta_{2 k+1}}^{\mathbf{0}}\left(Y_{2 k+1}\right)$.

2.4. The odd case. In the previous subsection we witnessed the construction of a Chern character for cohomotopy twists, which gave us a mapping from the higher twisted K-theory of a smooth compact manifold to its twisted cohomology. Strictly speaking, we constructed an even Chern character that mapped even twisted K-theory to even twisted cohomology. The purpose of this subsection is to extend this construction to the odd case. Namely, we construct an odd Chern character that will map odd twisted K-theory to odd twisted cohomology.

We have already seen that $A u t\left(\mathscr{O}_{\infty} \otimes \mathscr{K}\right)$ acts on Fred $_{\mathscr{O}_{\infty} \otimes \mathscr{K}}$ via conjugation, see section 1 . Letting $\Omega$ Fred $_{\mathscr{O}_{\infty} \otimes \mathscr{K}}$ denote the smooth pointed loop space. In other words, an element $\gamma \in \Omega$ Fred $d_{\mathscr{O}_{\infty} \otimes \mathscr{K}}$ consists of a smooth pointed map $\gamma: S^{1} \rightarrow$ Fred $_{\mathscr{O}_{\infty} \otimes \mathscr{K}}$, where the base point of Fred $\mathscr{O}_{\infty} \otimes \mathscr{K}$ is the identity operator. We see that this action extends to an action on $\Omega$ Fred $\mathscr{O}_{\infty} \otimes \mathscr{K}$ in the pointwise fashion. In other words, given a continuous loop $\gamma: S^{1} \rightarrow$ Fred $_{\mathscr{O}_{\infty} \otimes \mathscr{K}}$ and an element 
$A \in \operatorname{Aut}\left(\mathscr{O}_{\infty} \otimes \mathscr{K}\right)$. We define $A \cdot \gamma: S^{1} \rightarrow \operatorname{Fred}_{\mathscr{O}_{\infty} \otimes \mathscr{K}}$ to be the loop $(A \cdot \gamma)(t):=A(\gamma(t))$, where $A(\gamma(t))$ is the conjugation action of $A$ on the point $\gamma(t) \in$ Fred $_{\mathscr{O}_{\infty} \otimes \mathscr{K}}$. We point out that given an $\operatorname{Aut}\left(\mathscr{O}_{\infty} \otimes \mathscr{K}\right)$-bundle we can, via the above action and the associated bundle construction, produce an $\Omega$ Fred $\mathscr{O}_{\infty} \otimes \mathscr{K}$-bundle.

As we saw in section 2.1, the generator $u_{2 k+1} \in H^{2 k+1}\left(S^{2 k+1} ; \mathbb{Z}\right)$ gives rise to a principal $A u t\left(\mathscr{O}_{\infty} \otimes\right.$ $\mathscr{K}$ )-bundle via corollary 4.7 (ii) in [21]. Using this bundle, and the above action on $\Omega$ Fred $\mathscr{O}_{\infty} \otimes \mathscr{K}$, the associated bundle construction produces a $\Omega$ Fred $\mathscr{\mathscr { O }}_{\infty} \otimes \mathscr{K}$-bundle over $S^{2 k+1}$ corresponding to $u_{2 k+1}$, which we shall denote by $L_{2 k+1}$.

There is an evaluation map $e: \Omega$ Fred $_{\mathscr{O}_{\infty} \otimes \mathscr{K}} \times S^{1} \rightarrow$ Fred $_{\mathscr{O}_{\infty} \otimes \mathscr{K}}$ defined by $e(\gamma, t)=\gamma(t)$.

Given a cohomology generator $s_{n} \in H^{2 n}\left(\right.$ Fred $\left._{\mathscr{O}_{\infty} \otimes \mathscr{K}}\right)$ we let

$$
\Omega s_{n}:=\int_{S^{1}} e^{*} s_{n} \in H^{2 n-1}\left(\Omega \operatorname{Fred}_{\mathscr{O}_{\infty} \otimes \mathscr{K}}\right) .
$$

The above evaluation map induces an evaluation map, which we also denote by $e$

$$
e: L_{2 k+1} \times S^{1} \rightarrow Y_{2 k+1}
$$

as the reader can check.

We recall from section 2.2 that we constructed forms $S_{n} \in \Omega^{2 n}\left(Y_{2 k+1}\right)$. We define $T_{n}:=e^{*} S_{n} \in$ $\Omega^{2 n}\left(L_{2 k+1} \times S^{1}\right)$. Integrating over the fibre, we define forms

$$
\Omega S_{n}:=\int_{S^{1}} T_{n} \in \Omega^{2 n-1}\left(L_{2 k+1}\right) .
$$

We also recall that the forms $S_{n}$ satisfied the formula $d S_{n}=\frac{(-1)^{k+1} n !}{(n-k) !} \eta_{2 k+1} S_{n-k}$, where the form $\eta_{2 k+1}=m \eta$ is a $2 k+1$-form on $Y_{2 k+1}$ coming from the pull-back of a certain generating $2 k+1$ form on $S^{2 k+1}$, see section 2.2 for details.

The $2 k+1$-form $\eta_{2 k+1}$ is constant along the fibres of $Y_{2 k+1}$, as it comes from the pull-back of a form on the base via the projection map. In particular, since the evaluation map is fibre preserving, it follows that the form $e^{*}\left(\eta_{2 k+1}\right)$ does not have any $d \theta$-components, $\theta$ being a coordinate on $S^{1}$.

Proposition 2.6. $d \Omega S_{n}=\lambda(n, k) e^{*}\left(\eta_{2 k+1}\right) \Omega S_{n-k}$, where $\lambda(n, k):=\frac{(-1)^{k+1} n !}{(n-k) !}$. 
Proof. We differentiate under the integral, which is possible as $S^{1}$ is compact and all our forms are smooth.

$$
\begin{aligned}
d \Omega S_{n} & =d \int_{S^{1}} e^{*} S_{n} \\
& =\int_{S^{1}} d e^{*} S_{n} \\
& =\int_{S^{1}} e^{*} d S_{n} \\
& =\int_{S^{1}} e^{*}\left(\frac{(-1)^{k+1} n !}{(n-k) !} \eta_{2 k+1} S_{n-k}\right) \\
& =\int_{S^{1}} \frac{(-1)^{k+1} n !}{(n-k) !} e^{*}\left(\eta_{2 k+1}\right) e^{*}\left(S_{n-k}\right) \\
& =\frac{(-1)^{k+1} n !}{(n-k) !} e^{*}\left(\eta_{2 k+1}\right) \Omega S_{n-k}
\end{aligned}
$$

where the last equality comes from the fact that $e^{*}\left(\eta_{2 k+1}\right)$ will not have any $d \theta$-components, and so does not contribute to the integral.

We now define a Chern character for $L_{2 k+1}$, that will represent the universal odd Chern character for cohomotopy twists of degree $2 k+1$, by

$$
C h_{2 k+1}^{\mathbf{1}}=\sum \frac{\lambda(n, k) \Omega S_{n}}{n !} .
$$

It is easy to see that $D_{e^{*}\left(\eta_{2 k+1}\right)} C h_{2 k+1}^{\mathbf{1}}=\left(d-e^{*}\left(\eta_{2 k+1}\right)\right) C h_{2 k+1}^{\mathbf{1}}=0$. Hence $C h_{2 k+1}^{\mathbf{1}}$ is an element of the odd twisted cohomology $H_{e^{*}\left(\eta_{2 k+1}\right)}^{*}\left(L_{2 k+1}\right)$.

Given the odd Chern character for $L_{2 k+1}$, we can define a general odd Chern character for any space $X$ with a degree $2 k+1$-cohomotopy twist. This will be a map from the odd higher twisted K-theory of $X$ to twisted cohomology.

Given a smooth compact manifold $X$ with a cohomotopy class represented by a smooth map $\lambda: X \rightarrow S^{2 k+1}$, we observe that $\Omega$ Fred $\lambda(X):=\lambda^{*} L_{2 k+1}$ is a $\Omega$ Fred $\mathscr{O}_{\infty} \otimes \mathscr{K}$-bundle over $X$. Furthermore, the isomorphism class of this bundle over $X$ depends on the homotopy class of $\lambda$. This allows us to define the odd twisted K-theory of $X$, corresponding to the twist $\lambda$, in a similar way to the even case done in section 2.3.

Definition 2.7. Given a smooth compact manifold $X$, and a cohomotopy class $[\lambda] \in\left[X, S^{2 k+1}\right]$ represented by a smooth map $\lambda: X \rightarrow S^{2 k+1}$, we define the odd higher twisted $K$-theory of $X$, twisted by the cohomotopy class $[\lambda]$ and denoted $K_{\lambda}^{1}(X)$, to consist of homotopy classes in $\left[X, L_{2 k+1}\right]$ that lift the map $\lambda$. In other words, an element of $K_{\lambda}^{1}(X)$ is a homotopy class of a map $F: X \rightarrow L_{2 k+1}$ such that the following digram commutes

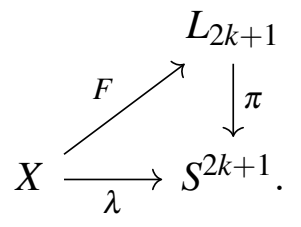


We remark that an equivalent way to define $K_{\lambda}^{\mathbf{1}}(X)$ would be as homotopy classes of sections of the bundle $\Omega$ Fred $\lambda(X)$. This follows from the fact that $\Omega$ Fred $d_{\lambda}(X)=\lambda^{*} L_{2 k+1}$. Furthermore, as Fred $_{\mathscr{O}_{\infty} \otimes \mathscr{K}}$ is a smooth Banach manifold, it follows that $\Omega$ Fred $\mathscr{O}_{\infty} \otimes \mathscr{K}$ is also a smooth Banach manifold. This implies the bundle $L_{2 k+1}$ can be given the structure of a smooth Banach manifold, and hence the concept of a smooth map from $X$ to $L_{2 k+1}$ is well defined.

We define the odd twisted Chern character

$$
C h_{\lambda}^{1}: K_{\lambda}^{o d d}(X) \rightarrow H_{\lambda *\left(u_{2 k+1}\right)}^{1}(X)
$$

by $C h_{\lambda}^{\mathbf{1}}([F])=\left[F^{*} C h_{\lambda}^{\mathbf{1}}\right]$. We remind the reader that when we form the pull back differential form $F^{*} C h_{\lambda}^{1}$, we always take a smooth representative $F$. The fact that this defines an element of the odd twisted cohomology group $H_{\lambda^{*}\left(u_{2 k+1}\right)}(X)$ follows by naturality and the fact that $C h_{2 k+1}^{\mathbf{1}} \in$ $H_{e^{*}\left(\eta_{2 k+1}\right)}^{\mathbf{1}}\left(L_{2 k+1}\right)$.

\section{SPHERICAL T-DUALity}

3.1. Historical introduction. We begin with a historical introduction.

T-duality for pairs consisting of a circle bundle, together with a degree $3 \mathrm{H}$-flux, was originally studied in detail in $[11,12]$ with contributions by several others later. In string theory, T-dual pairs are distinct compactification manifolds that cannot be distinguished by any experiment, which is the notion of isomorphism relevant in physics. This equivalence in physics implies the isomorphisms of a number of other mathematical structures, such as Courant algebroids [18], generalized complex structures [18] and twisted K-theory [11], see also [17, 44]. It turns out that all of these structures are physically relevant.

Recently we initiated the study of spherical T-duality for principal $S U$ (2)-bundles in [13] and for non-principal $S U$ (2)-bundles in [14], together with further insights in [15]. Let $P$ be a principal $S U$ (2)-bundle over $M$ and $H$ a 7-cocycle on $P$,

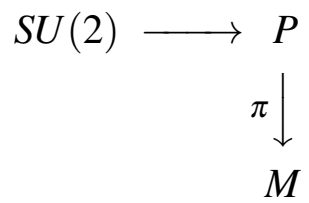

Principal $S U$ (2)-bundles over a compact oriented four dimensional manifold $M$ are classified by $H^{4}(M ; \mathbb{Z}) \cong \mathbb{Z}$ via the 2 nd Chern class $c_{2}(P)$. This can be seen using the well known isomorphism, $H^{4}(M ; \mathbb{Z}) \cong\left[M, S^{4}\right] \cong \mathbb{Z}$ and noting that there is a canonical principal $S U(2)$-bundle $P \rightarrow S^{4}$, known as the Hopf bundle, whose 2 nd Chern class is the generator of $H^{4}\left(S^{4} ; \mathbb{Z}\right) \cong \mathbb{Z}$. The orientation of $M$ and $S U(2)$ imply that $\pi_{*}$ is a canonical isomorphism $H^{7}(P ; \mathbb{Z}) \cong H^{4}(M ; \mathbb{Z}) \cong \mathbb{Z}$. The dual principal $S U(2)$-bundle,

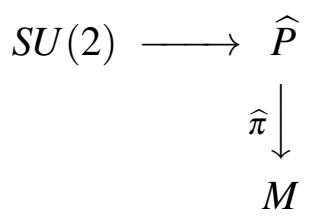

is defined by $c_{2}(\widehat{P})=\pi_{*} H$ while the dual 7-cocycle $\widehat{H} \in H^{7}(\widehat{P})$ satisfies $c_{2}(P)=\widehat{\pi}_{*} \widehat{H}$ by the isomorphism $\widehat{\pi}_{*}: H^{7}(\widehat{P} ; \mathbb{Z}) \cong H^{4}(M ; \mathbb{Z}) \cong \mathbb{Z}$. We proved that this spherical T-duality map induces 
degree-shifting isomorphisms between the real and integral twisted cohomologies of $P$ and $\widehat{P}$ and also between the 7-twisted K-theories.

In [14], we extended spherical T-duality to (oriented) non-principal $S U(2)$-bundles. While principal $S U$ (2)-bundles correspond to unit sphere bundles of quaternionic line bundles, (oriented) non-principal $S U$ (2)-bundles correspond to unit sphere bundles of rank 4 oriented real Riemannian vector bundles. A striking new phenomenon in the non-principal case is that when the base $M$ is a compact oriented simply-connected 4 dimensional manifold, and given an oriented nonprincipal $S U$ (2)-bundle $E$ with $H$ a 7-cocycle on $E$, then for each integer, we will show that there is an infinite lattice of spherical T-duals with 7-cocycle flux over $M$, in stark contrast to the case of principal $S U$ (2)-bundles as described above. One reason is because in the non-principal bundle case, the Euler class does not determine (oriented) non-principal $S U$ (2)-bundles when the base is as above, but in addition the 2nd Stiefel-Whitney class and the Pontryagin class are also needed for the classification. However, it is only the Euler class (and its transgression) that is needed in the Gysin sequence and also to prove the isomorphisms of 7-twisted integral cohomologies, and in addition the 2nd Stiefel-Whitney class is needed to prove the isomorphisms of 7-twisted K-theories.

3.2. Spherical T-duality computations. Let $M$ be a compact, oriented manifold of dimension $2 n$. Since in high dimensions, the classification up to isomorphism of $S^{2 n-1}$ bundles over $M$ by cohomology is complicated, or unknown, we only consider the Euler characteristic of such bundles. In general there may be several bundles (over a fixed base $M$ ) that have the same Euler characteristic, but they all have the same cohomology groups and the same K-theory groups, as we will show.

3.3. Spherical T-dual pairs. Here we define spherical T-dual pairs over $M$ as above. Suppose that $n=3$ or $n>4$. Consider the sphere bundle $Z$ over $M$ with structure group $S O(2 n)$

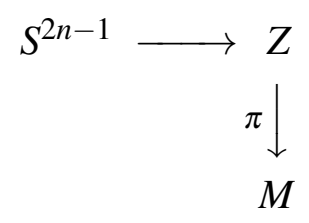

Then it has an Euler class $e(Z) \in H^{2 n}(M, \mathbb{Z})$ which is an even integer $2 k$ ([48]). Let $H$ be a degree $(4 n-1)$-form over $Z$ such that $[H] \in H^{4 n-1}(Z, \mathbb{Z})$ is the even integer $2 l$. Then $(Z, H)$ has a spherical T-dual $(\widehat{Z}, \widehat{H})$ defined as follows.

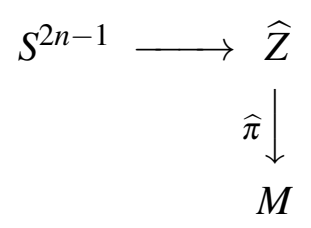

It has Euler class $e(\widehat{Z})=\pi_{*}(H) \in H^{2 n}(M, \mathbb{Z})$ that is equal to $2 l$. Since $\widehat{\pi}_{*}: H^{4 n-1}(\widehat{Z}, \mathbb{Z}) \longrightarrow$ $H^{2 n}(M, \mathbb{Z})$ is an isomorphism, we define $\widehat{H}=\widehat{\pi}_{*}^{-1} \pi_{*}(H)$.

To summarise, $(Z, H)$ and $(\widehat{Z}, \widehat{H})$ are defined to be spherical T-dual pairs over $M$. 
Now we finish by defining spherical T-dual pairs over $M$ when $n=4$ i.e. an 8 dimensional manifold as above. Consider the special sphere bundle $Z$ over $M$ with structure group $S O(8)$

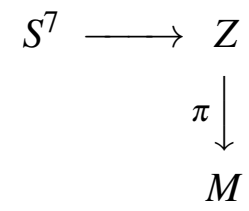

Then the Euler class $e(Z) \in H^{8}(M, \mathbb{Z})$ can be any integer $k$, since there is a Hopf bundle over $S^{8}$. Let $H$ be a 15 -form over $Z$ such that $[H] \in H^{15}(Z, \mathbb{Z})$ is the integer $l$. Then $(Z, H)$ has a spherical T-dual $(\widehat{Z}, \widehat{H})$ defined as follows.

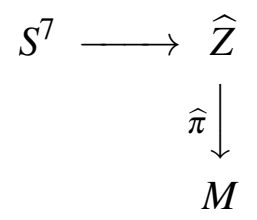

where the Euler class $e(\widehat{Z})=\pi_{*}(H) \in H^{4}(M, \mathbb{Z})$ is equal to $l$. Since $\widehat{\pi}_{*}: H^{15}(\widehat{Z}, \mathbb{Z}) \longrightarrow H^{8}(M, \mathbb{Z})$ is an isomorphism, we define $\widehat{H}=\widehat{\pi}_{*}^{-1} \pi_{*}(H)$ which equals $k$.

To summarise, $(Z, H)$ and $(\widehat{Z}, \widehat{H})$ are spherical $T$-dual pairs over $M$.

3.4. Gysin sequence and the cohomology of $Z$ and $\widehat{Z}$. Since $Z$ is a sphere bundle over $M$ for $n=3$ or for $n>4$, with Euler class $e(Z)=2 k$, there are Gysin sequences that will compute the cohomology of $Z$. Assume here that $k \neq 0$. The Gysin sequence is

$\rightarrow H^{j}(M, \mathbb{Z}) \stackrel{\pi^{*}}{\rightarrow} H^{j}(Z, \mathbb{Z}) \stackrel{\pi_{*}}{\rightarrow} H^{j-(2 n-1)}(M, \mathbb{Z}) \stackrel{\cup 2 k}{\rightarrow} H^{j+1}(M, \mathbb{Z}) \rightarrow H^{j+1}(Z, \mathbb{Z}) \stackrel{\pi_{*}}{\rightarrow} H^{j+2-2 n}(M, \mathbb{Z}) \rightarrow$

It follows that

$$
\begin{aligned}
& H^{j}(Z, \mathbb{Z}) \cong H^{j}(M, \mathbb{Z}), \quad 0 \leq j<2 n-1 \text {,i.e. } \\
& H^{0}(Z, \mathbb{Z})=\mathbb{Z}, H^{j}(Z, \mathbb{Z})=H^{j}(M, \mathbb{Z}) \quad \text { for } \quad 0 \leq j \leq 2 n-1 \\
& H^{2 n}(Z, \mathbb{Z})=\mathbb{Z}_{2 k} \\
& H^{j}(Z, \mathbb{Z})=H^{j+1-2 n}(M, \mathbb{Z}), \quad 2 n<j<4 n-2 \\
& H^{4 n-1}(Z, \mathbb{Z})=\mathbb{Z} \text {. } \\
& H^{\text {odd }}(Z, \mathbb{Z}) \cong \mathbb{Z} \bigoplus_{j=1}^{7} H^{j}(M, \mathbb{Z}) . \\
& H^{\text {even }}(Z, \mathbb{Z}) \cong \mathbb{Z} \oplus \mathbb{Z}_{2 k} \bigoplus_{j=1}^{2 n-1} H^{j}(M, \mathbb{Z}) \\
& H^{\text {odd }}(Z, \mathbb{Z}) \cong \mathbb{Z} \bigoplus_{j=1}^{2 n-1} H^{j}(M, \mathbb{Z}) \text {. }
\end{aligned}
$$


When $k=0$, then we get that

$$
\begin{aligned}
& H^{\text {even }}(Z, \mathbb{Z}) \cong \mathbb{Z} \oplus \mathbb{Z} \bigoplus_{j=1}^{2 n-1} H^{j}(M, \mathbb{Z}) \\
& H^{\text {odd }}(Z, \mathbb{Z}) \cong \mathbb{Z} \bigoplus_{j=1}^{2 n-1} H^{j}(M, \mathbb{Z}) .
\end{aligned}
$$

Similarly for $S^{7}$-bundles $Z$ over $M^{8}$ with Euler class $k$, repeating the calculations above, we see that for $k \neq 0$,

$$
\begin{aligned}
& H^{\text {even }}(Z, \mathbb{Z}) \cong \mathbb{Z} \oplus \mathbb{Z}_{k} \bigoplus_{j=1}^{7} H^{j}(M, \mathbb{Z}) \\
& H^{\text {odd }}(Z, \mathbb{Z}) \cong \mathbb{Z} \bigoplus_{j=1}^{7} H^{j}(M, \mathbb{Z}) .
\end{aligned}
$$

When $k=0$ we get that

$$
\begin{aligned}
& H^{\text {even }}(Z, \mathbb{Z}) \cong \mathbb{Z} \oplus \mathbb{Z} \bigoplus_{j=1}^{7} H^{j}(M, \mathbb{Z}) \\
& H^{\text {odd }}(Z, \mathbb{Z}) \cong \mathbb{Z} \bigoplus_{j=1}^{7} H^{j}(M, \mathbb{Z})
\end{aligned}
$$

3.5. Isomorphism of higher twisted cohomologies. We use the calculations above to now compute the higher twisted cohomology groups $H_{H}^{\text {even } / o d d}(Z, \mathbb{Z})$, computed via the secondary operation $\cup H$ acting on $H^{\bullet}(Z, \mathbb{Z})$. Note that $(\cup H)^{2}=0$ for degree reasons.

$\cup H: H^{0}(Z, \mathbb{Z})=\mathbb{Z} \longrightarrow H^{4 n-1}(Z, \mathbb{Z})=\mathbb{Z}$ is multiplication by $2 l$, and since $l \neq 0$, the nullspace is zero and the image is $2 l \mathbb{Z}$. It follows that the twisted cohomology groups are

$$
\begin{gathered}
H_{H}^{\text {even }}(Z, \mathbb{Z}) \cong \mathbb{Z}_{2 k} \bigoplus_{j=1}^{2 n-1} H^{j}(M, \mathbb{Z}) \\
H_{H}^{\text {odd }}(Z, \mathbb{Z})=\cong \mathbb{Z}_{2 l} \bigoplus_{j=1}^{2 n-1} H^{j}(M, \mathbb{Z}) .
\end{gathered}
$$

Noting that $(Z, H)$ and $(\widehat{Z}, \widehat{H})$ are spherical $T$-dual pairs over $M$, where $e(\widehat{Z})=2 l$ and $\widehat{H}=2 k$, we see that

$$
\begin{aligned}
& H_{\widehat{H}}^{\text {even }}(\widehat{Z}, \mathbb{Z}) \cong \mathbb{Z}_{2 l} \bigoplus_{j=1}^{2 n-1} H^{j}(M, \mathbb{Z}) \\
& H_{\widehat{H}}^{\text {odd }}(\widehat{Z}, \mathbb{Z}) \cong \mathbb{Z}_{2 k} \bigoplus_{j=1}^{2 n-1} H^{j}(M, \mathbb{Z}) .
\end{aligned}
$$


We conclude that the spherical T-duality isomorphisms hold:

$$
\begin{aligned}
H_{H}^{\text {even }}(Z, \mathbb{Z}) & \left.\cong H_{\widehat{H}}^{\text {odd }}(\widehat{Z}, \mathbb{Z})\right) \\
H_{H}^{\text {odd }}(Z, \mathbb{Z}) & \cong H_{\widehat{H}}^{\text {even }}(\widehat{Z}, \mathbb{Z}) .
\end{aligned}
$$

The same is true when either $k=0$ or $l=0$.

For $S^{7}$-bundles $Z$ over $M=M^{8}$ with Euler class $k$ and degree 15 -flux $H$ equal to $l$, we can calculate analogous to the above that $H_{H}^{\text {even }}(Z, \mathbb{Z})=\mathbb{Z}_{k} \bigoplus_{j=1}^{7} H^{j}(M, \mathbb{Z})$ and $H_{H}^{\text {odd }}(Z, \mathbb{Z})=\mathbb{Z}_{l} \bigoplus_{j=1}^{7} H^{j}(M, \mathbb{Z})$ giving the spherical $T$ duality isomorphisms

$$
\begin{aligned}
H_{H}^{\text {even }}(Z, \mathbb{Z}) & \left.\cong H_{\widehat{H}}^{\text {odd }}(\widehat{Z}, \mathbb{Z})\right) \\
H_{H}^{\text {odd }}(Z, H, \mathbb{Z}) & \cong H_{\widehat{H}}^{\text {even }}(\widehat{Z}, \mathbb{Z}) .
\end{aligned}
$$

3.6. K-theory of $Z$ and $\widehat{Z}$ when the base $M$ is torsion free. In this subsection, we will prove that the even K-theory of $Z$ is isomorphic to the even cohomology of $Z$, and that the odd K-theory of $Z$ is isomorphic to the odd cohomology of $Z$, under the added assumption that the base manifold $M$ is torsion free. Similar results hold for the $T$-dual $\widehat{Z}$.

As we will be applying the Gysin sequence, which depends on the $\mathbb{Z}$ grading of the $K$-theory groups, we will have to consider the $\mathbb{Z}$ graded $K$-groups $K^{j}(\cdot)$ for $j \in \mathbb{Z}$. By Bott periodicity we know that these groups are 2 periodic. Therefore, we will let $K^{\text {even }}(\cdot)$ denote the even $K$-groups and $K^{o d d}(\cdot)$ the odd $K$-groups. While this notation is slightly different to the one employed in section 2 , it is employed so as to not cause confusion with the $K^{0}$ and $K^{1}$ terms that arise in the Gysin sequence. In the case of cohomology, we follow the notation used in the previous subsection. That is, $H^{\text {even }}(\cdot ; \mathbb{Z})$ will denote the direct sum of all even cohomology groups, and $H^{\text {odd }}(\cdot ; \mathbb{Z})$ the direct sum of all the odd cohomology groups.

Using the Gysin sequence in $K$-theory, we obtain

$$
\begin{gathered}
\cdots \longrightarrow K^{j-2 n}(M) \stackrel{\phi}{\longrightarrow} K^{j}(M) \stackrel{\pi^{*}}{\longrightarrow} K^{j}(Z) \stackrel{\pi_{*}}{\longrightarrow} K^{j-(2 n-1)}(M) \\
\longrightarrow K^{j+1}(M) \stackrel{\pi^{*}}{\longrightarrow} K^{j+1}(Z) \stackrel{\pi_{*}}{\longrightarrow} K^{j+(2 n-1)}(M) \longrightarrow \cdots \cdots
\end{gathered}
$$

The map $\phi$ is the $K$-theoretic version of cupping with the Euler class, and is defined on the even $K$-groups by $\phi(x)=r k(x) k \cdot T h$, with $T h$ being the Thom class and $r k$ being the rank function. On the odd $K$-groups $\phi \equiv 0$.

As we are assuming $M$ is torsion free, it follows that $K^{\text {even }}(M) \cong H^{\text {even }}(M ; \mathbb{Z})$. This follows from a simple calculation using the Atiyah-Hirzebruch spectral sequence, and the fact that all differentials in this spectral sequence are torsion operators, see [1]. Cupping with the Euler class is a map from $H^{0}(M ; \mathbb{Z}) \cong \mathbb{Z}$ to $H^{2 n}(M ; \mathbb{Z}) \cong \mathbb{Z}$, given by multiplication by $2 k$. Under the isomorphism $K^{\text {even }}(M) \cong H^{\text {even }}(M ; \mathbb{Z})$, the map $\phi$ has image

$$
2 k \mathbb{Z} \subseteq \mathbb{Z} \cong H^{2 n}(M ; \mathbb{Z}) \subseteq H^{\text {even }}(M ; \mathbb{Z}) .
$$

We further observe that when $j$ is even, the kernel of $\phi$ is the reduced $K$-group $\widetilde{K}^{j-2 n}(M)$. The fact that $K^{\text {even }}(M) \cong H^{\text {even }}(M ; \mathbb{Z})$ then gives $\operatorname{ker} \phi=\widetilde{K}^{j-2 n}(M) \cong \bigoplus_{j=1}^{n} H^{2 j}(M ; \mathbb{Z})$. 
We start by analysing the even $K$-groups of $Z$. When $j=0$, we can extract out the following sequence using the fact that $\phi \equiv 0$.

$$
\begin{gathered}
0 \longrightarrow K^{-1}(M) \stackrel{\pi^{*}}{\longrightarrow} K^{-1}(Z) \stackrel{\pi_{*}}{\longrightarrow} K^{-2 n}(M) \stackrel{\phi}{\longrightarrow} K^{0}(M) \stackrel{\pi^{*}}{\longrightarrow} K^{0}(Z) \\
\rightarrow K^{-(2 n-1)}(M) \stackrel{\phi}{\longrightarrow} 0
\end{gathered}
$$

From this sequence we can extract two short exact sequences. The first exact sequence being

$$
0 \longrightarrow \operatorname{ker} \pi^{*}=\operatorname{im} \phi \longrightarrow K^{0}(M) \stackrel{\pi^{*}}{\longrightarrow} \operatorname{im} \pi^{*} \stackrel{\phi}{\longrightarrow} 0
$$

which gives $\operatorname{im} \pi^{*} \cong \frac{K^{0}(M)}{\operatorname{im} \phi} \cong \frac{K^{0}(M)}{2 k \mathbb{Z}}$.

As $K^{0}(M) \cong H^{\text {even }}(M ; \mathbb{Z})$, and im $\phi$ can be identified with $2 k \mathbb{Z} \subseteq H^{2 n}(M ; \mathbb{Z})$, we see that

$$
\operatorname{im} \pi^{*} \cong \bigoplus_{j=0}^{n-1} H^{2 j}(M ; \mathbb{Z}) \oplus \mathbb{Z}_{2 k}
$$

The second exact sequence we obtain is

$$
0 \longrightarrow \operatorname{ker} \pi_{*}=\operatorname{im} \pi^{*} \longrightarrow K^{0}(Z) \stackrel{\pi_{*}}{\longrightarrow} K^{-(2 n-1)}(M) \stackrel{\phi}{\longrightarrow} 0
$$

The torsion free condition on $M$ implies $K^{2 n-1}(M) \cong H^{\text {odd }}(M ; \mathbb{Z})$, which is a torsion free abelian group. Hence, the above short exact sequence splits and, on applying Bott periodicity, we obtain

$$
K^{\text {even }}(Z) \cong \bigoplus_{j=0}^{n-1} H^{2 j}(M ; \mathbb{Z}) \oplus \mathbb{Z}_{2 k} \oplus H^{\text {odd }}(M ; \mathbb{Z}) .
$$

The odd $K$-groups of $Z$ can be analysed in a similar manner. Letting $j=1$ in the $K$-theoretic Gysin sequence, and using the fact that $\phi \equiv 0$ on the odd $K$-groups, gives the following sequence

$$
\begin{gathered}
0 \stackrel{\text { ․ }}{\longrightarrow} K^{1}(M) \stackrel{\pi^{*}}{\longrightarrow} K^{1}(Z) \stackrel{\pi_{*}}{\longrightarrow} K^{2-2 n}(M) \stackrel{\phi}{\longrightarrow} K^{2}(M) \stackrel{\pi^{*}}{\longrightarrow} K^{2}(Z) \\
\longrightarrow K^{3-2 n}(M) \stackrel{\phi}{\longrightarrow} 0
\end{gathered}
$$

From this exact sequence we can extract the short exact sequence

$$
0 \longrightarrow K^{1}(M) \stackrel{\pi^{*}}{\longrightarrow} K^{1}(Z) \stackrel{\pi_{*}}{\longrightarrow} \operatorname{im} \pi_{*}=\operatorname{ker} \phi \longrightarrow 0
$$

As $\operatorname{ker} \phi \cong \bigoplus_{j=1}^{n} H^{2 j}(M ; \mathbb{Z})$ is a free abelian group, the above short exact sequence splits. Applying Bott periodicity, we thus obtain

$$
K^{\text {odd }}(Z) \cong K^{1}(M) \oplus \bigoplus_{j=1}^{n} H^{2 j}(M ; \mathbb{Z}) \cong H^{\text {odd }}(M ; \mathbb{Z}) \oplus \bigoplus_{j=1}^{n} H^{2 j}(M ; \mathbb{Z}) .
$$

These observations allow us to prove the following theorem.

Theorem 3.1. $K^{\text {even } / \text { odd }}(Z) \cong H^{\text {even } / \text { odd }}(Z ; \mathbb{Z})$. 
Proof. The above discussion shows $K^{\text {even }}(Z) \cong \bigoplus_{j=0}^{n-1} H^{2 j}(M ; \mathbb{Z}) \oplus Z / 2 k \mathbb{Z} \oplus H^{\text {odd }}(M ; \mathbb{Z}) \cong H^{\text {even }}(Z ; \mathbb{Z})$. This last isomorphism coming from the Gysin sequence computation in section 3.4.

Similarly, using the cohomology Gysin sequence computation for $H^{\text {odd }}(Z ; \mathbb{Z})$, carried out in section 3.4, and the above computation for $K^{o d d}$, proves the odd case.

A similar result holds for the $T$-dual $\widehat{Z}$. As the proof is exactly analogous to what was done above for $Z$, we simply state the final theorem.

Theorem 3.2. $K^{\text {even } / \text { odd }}(\widehat{Z}) \cong H^{\text {even } / \text { odd }}(\widehat{Z} ; \mathbb{Z})$.

3.7. Isomorphism of higher Twisted $K$-theories when the base $M$ is torsion free. In this subsection we compute the higher twisted K-theory of $Z$ and $\widehat{Z}$, and show that they are isomorphic after a degree shift. This should be compared with similar results obtained for the twisted cohomologies in section 3.5. As in the previous subsection, we will be assuming that $M$ is torsion free. We remind the reader that $Z$ has Euler class $e(Z) \in H^{2 n}(M ; \mathbb{Z})$ corresponding to the integer $2 k$. We also fixed a top degree cohomology class $H \in H^{4 n-1}(Z ; \mathbb{Z})$, that corresponds to the integer $2 l$. The spherical $T$-dual $\widehat{Z}$ has Euler class $e(\widehat{Z})=2 l$. Furthermore, we have fixed $\widehat{H} \in H^{4 n-1}(\widehat{Z} ; \mathbb{Z})$ given by $\widehat{H}=2 k$. We will be computing the twisted K-theory of $Z$, twisted by the class $H$ and denoted by $K_{H}^{\mathbf{0}}$ and $K_{H}^{\mathbf{1}}$, as well as the twisted K-theory of $\widehat{Z}$, twisted by the class $\widehat{H}$. It is these twisted $K$-theory groups that will be shown to be isomorphic after a degree shift. We will do this via the Atiyah-Hirzebruch spectral sequence for twisted $K$-theory. 


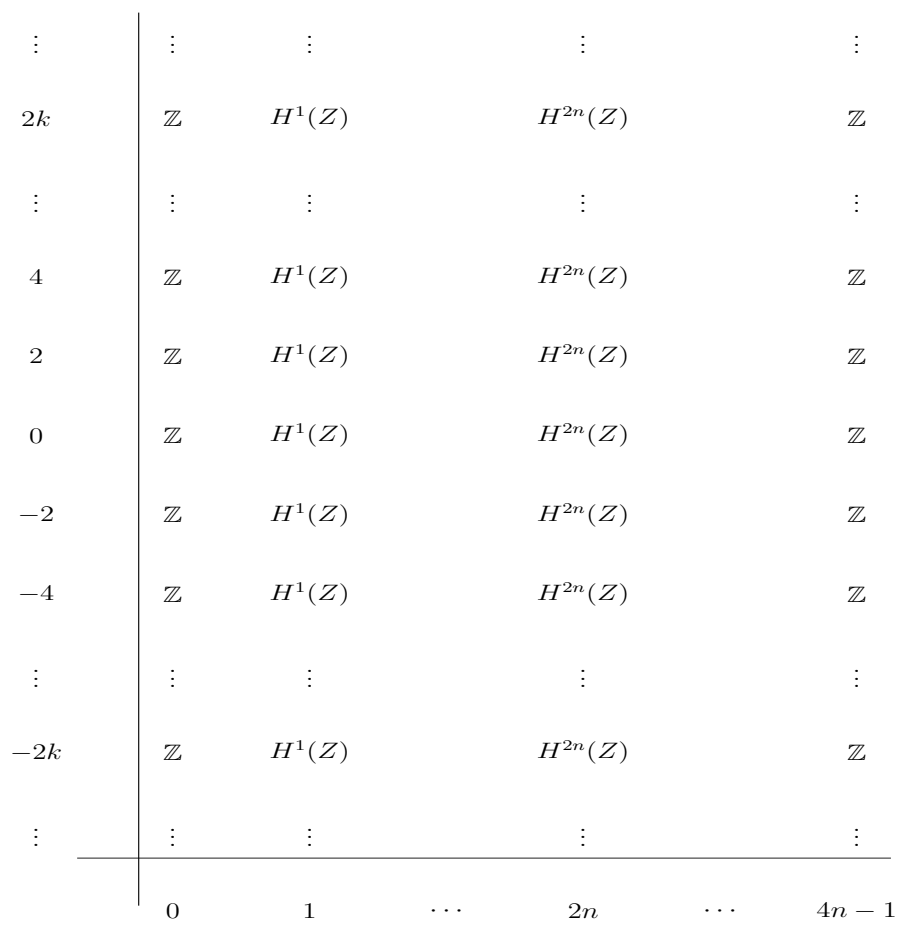

Therefore, the $E_{2}$-page of the spectral sequence (as in the figure) takes the following form. The $E_{2}$-page of the Atiyah-Hirzebruch spectral sequence for $K_{H}^{*}$ is given by $E_{2}^{p, q}=H^{p}\left(Z ; K^{q}(p t)\right.$, where $K$ denotes ordinary complex $K$-theory. As $K^{o d d}(p t)=0$ and $K^{\text {even }}(p t)=\mathbb{Z}$, we see that $E_{2}^{p, 2 j}=H^{p}(Z ; \mathbb{Z})$ and $E_{2}^{p, 2 j+1}=0$ for all $j \in \mathbb{Z}$.

From the spectral sequence we can see that the even differentials all vanish. In the untwisted case, (i.e. when $H=0$ ) it is known that the odd differentials are all torsion operators, see [1]. It follows that the odd differentials $d_{j}$ for $j<4 n-1$ are all torsion operators, as these are equal to the differentials in the untwisted case. As $Z$ only has torsion in $H^{2 n}(Z ; \mathbb{Z})$, we see that the potential non-zero differentials are $d_{3}, d_{5}, \cdots, d_{2 n-1}$ and $d_{4 n-1}$.

Proposition 3.3. The differentials $d_{3}, d_{5}, \cdots, d_{2 n-1}$ are all zero.

Proof. The projection $\pi: Z \rightarrow M$ induces a map from the Atiyah-Hirzebruch spectral sequence of $K^{*}(M)$ to the Atiyah-Hirzebruch spectral sequence of $K^{*}(P)$. 
As the $E_{3}$-page of the Atiyah-Hirzebruch spectral sequence is the $E_{2}$-page, it follows that the induced map on the $E_{3}$-page, induced by $\pi$, is $\pi^{*}$ and this commutes with $d_{3}: H^{2 n-3}(Z ; \mathbb{Z}) \rightarrow$ $H^{2 n}(Z ; \mathbb{Z})$. We have already seen in section 3.4 that $\pi^{*}: H^{j}(M ; \mathbb{Z}) \rightarrow H^{j}(Z ; \mathbb{Z})$ is an isomorphism for $j<2 n$. If we take $x \in H^{2 n-3}(Z ; \mathbb{Z})$ let $y \in H^{2 n-3}(M ; \mathbb{Z})$ satisfy $\pi^{*}(y)=x$. We then see that $d_{3}(x)=d_{3} \circ \pi^{*}(y)=\pi^{*} \circ d_{3}(y)=0$. This latter equality following from the fact that $H^{2 n}(M ; \mathbb{Z}) \cong \mathbb{Z}$ and $d_{3}$ being a torsion operator. Thus we have proved that $d_{3} \equiv 0$.

The general case proceeds by induction. Assume that the differentials $d_{2 j-1} \equiv 0$ for all $2 \leq j \leq$ $i-1<n$. We will prove that $d_{2 i-1} \equiv 0$.

The induction hypothesis tells us that the induced map on the $E_{2 i-1}$-page, induced by $\pi$, is still $\pi^{*}$ and that this map commutes with $d_{2 i-1}$. Given $x \in H^{2 n-(2 i-1)}(Z ; \mathbb{Z})$, and using the fact that $\pi^{*}: H^{2 n-(2 i-1)}(M ; \mathbb{Z}) \rightarrow H^{2 n-(2 i-1)}(Z ; \mathbb{Z})$ is an isomorphism, let $y \in H^{2 n-(2 i-1)}(M ; \mathbb{Z})$ such that $\pi^{*}(y)=x$. We then have $d_{2 i-1}(x)=d_{2 i-1} \circ \pi^{*}(y)=\pi^{*} \circ d_{2 i-1}(y)=0$, where again we have used the fact that $d_{2 i-1}$ is a torsion operator and hence must vanish on $H^{2 n-(2 i-1)}(M ; \mathbb{Z})$.

We have proved that the differentials $d_{3}, d_{5}, \cdots, d_{2 n-1}$ are all zero in the Atiyah-Hirzebruch spectral sequence for the $K$-theory groups $K^{*}(Z)$. However, these differentials are also the differentials in the twisted Atiyah-Hirzebruch spectral sequence for $K_{H}^{*}(Z)$ in that range (the difference occurs for the differentials after $d_{4 n-3}$ ). Thus the theorem has been proved.

Proposition 3.4. The differential $d_{4 n-1}=\cup H=\cup 2 l$.

Proof. The cohomology class $H \in H^{4 n-1}(Z ; \mathbb{Z})$ is represented by a map $\widetilde{H}: Z \rightarrow K(\mathbb{Z}, 4 n-1)$, such that $\widetilde{H}^{*}(\eta)=H$, where $\eta$ is a generator of $H^{4 n-1}(K(\mathbb{Z}, 4 n-1) ; \mathbb{Z})$. As $Z$ has dimension $4 n-1$, we can apply the cellular approximation theorem, and assume the image of $\widetilde{H}$ is contained in the $4 n-1$-skeleton of $K(\mathbb{Z}, 4 n-1)$. The $4 n-1$-skeleton of $K(\mathbb{Z}, 4 n-1)$ is $S^{4 n-1}$, and therefore we have $\widetilde{H}: Z \rightarrow S^{4 n-1}$ with a generator $\eta \in H^{4 n-1}\left(S^{4 n-1} ; \mathbb{Z}\right)$ satisfying $\widetilde{H}^{*}(\eta)=H$.

The map $\widetilde{H}$ induces a map of the associated twisted Atiyah-Hirzebruch spectral sequences of $Z$ and $S^{4 n-1}$ respectively. Therefore, it suffices to prove the proposition for $S^{4 n-1}$ with twist given by the generator $\eta$. This latter case follows from the Mayer-Vietoris argument in [17].

Propositions 3.3 and 3.4 imply that the only non-zero differential in the Atiyah-Hirzebruch spectral sequence for $Z$ is $d_{4 n-1}: H^{0}(Z ; \mathbb{Z}) \cong \mathbb{Z} \rightarrow H^{4 n-1}(Z ; \mathbb{Z}) \cong \mathbb{Z}$. 


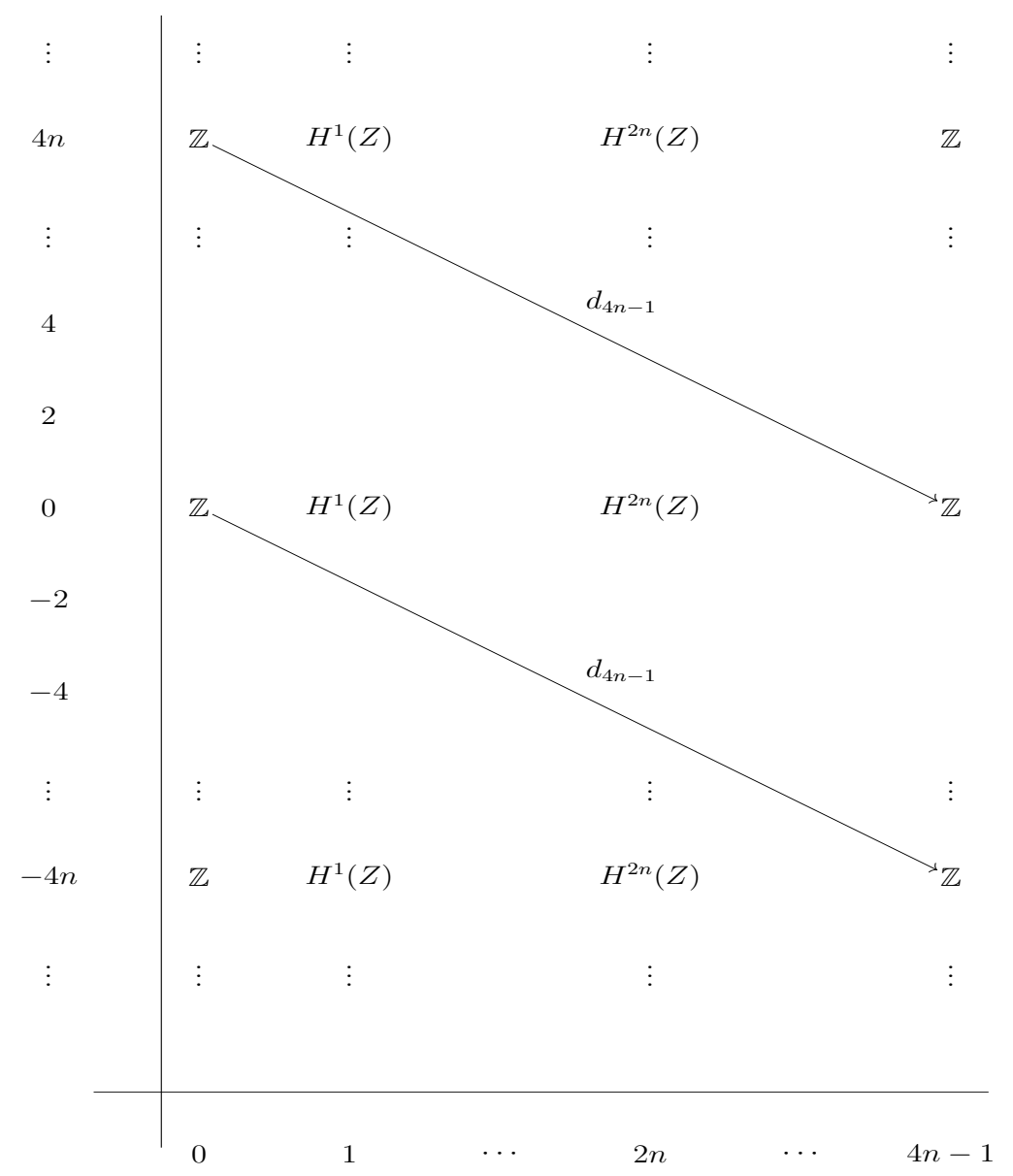

We thus find that the $E_{\infty}$-page is given in the following way: 


\begin{tabular}{|c|c|c|c|}
\hline$\vdots$ & $\vdots$ & $\vdots$ & $\vdots$ \\
\hline $2 k$ & $H^{1}(Z)$ & $H^{2 n}(Z)$ & $\mathbb{Z}_{2 l}$ \\
\hline$\vdots$ & $\vdots$ & $\vdots$ & $\vdots$ \\
\hline 4 & $H^{1}(Z)$ & $H^{2 n}(Z)$ & $\mathbb{Z}_{2 l}$ \\
\hline 2 & $H^{1}(Z)$ & $H^{2 n}(Z)$ & $\mathbb{Z}_{2 l}$ \\
\hline 0 & $H^{1}(Z)$ & $H^{2 n}(Z)$ & $\mathbb{Z}_{2 l}$ \\
\hline-2 & $H^{1}(Z)$ & $H^{2 n}(Z)$ & $\mathbb{Z}_{2 l}$ \\
\hline-4 & $H^{1}(Z)$ & $H^{2 n}(Z)$ & $\mathbb{Z}_{2 l}$ \\
\hline$\vdots$ & $\vdots$ & $\vdots$ & $\vdots$ \\
\hline$-2 k$ & $H^{1}(Z)$ & $H^{2 n}(Z)$ & $\mathbb{Z}_{2 l}$ \\
\hline$\vdots$ & $\vdots$ & $\vdots$ & $\vdots$ \\
\hline & 1 & $2 n$ & $4 n-1$ \\
\hline
\end{tabular}

From the $E_{\infty}$-page of the spectral sequence we can compute the twisted K-theory groups $K_{H}^{*}(Z)$. We do this via the filtration of the twisted K-theory groups. We remind the reader how this proceeds.

Given the sphere bundle $Z$, of dimension $4 n-1$, we fix a CW-complex structure on $Z$. Note that the CW-complex structure on $Z$ will have cells in dimensions $k \leq 4 n-1$, we will denote the $k^{t h}$-skeleton of $Z$ by $Z_{k}$. We then have the following filtration of $K_{H}^{n}(Z), n \in \mathbb{Z}$,

$$
K_{H}^{n}(Z) \supseteq \mathscr{F}^{0} \supseteq \mathscr{F}^{1} \supseteq \ldots \supseteq \mathscr{F}^{4 n-2} \supseteq \mathscr{F}^{4 n-1}=\{0\}
$$


where $\mathscr{F}^{i}:=\operatorname{ker}\left(K_{H}^{n}(Z) \rightarrow K_{H}^{n}\left(Z_{i}\right)\right)$, the map $K_{H}^{n}(Z) \rightarrow K_{H}^{n}\left(Z_{i}\right)$ being induced by the inclusion $Z_{i} \hookrightarrow Z$.

The convergence of the spectral sequence implies that $E_{\infty}^{p, q}=\frac{\mathscr{F}^{p-1}}{\mathscr{F}^{p}}$, where $p+q=n$. We refer the reader to the final section for a more detailed description of this spectral sequence.

The above $E_{\infty}$-page for $K_{H}^{*}(Z)$ differs from the untwisted complex K-theory $K^{*}(Z)$ in only the 0 and $4 n-1$ lines. As the 0 -line consists entirely of zeros, it follows that $K_{H}^{\mathbf{0}}(Z) \cong \widetilde{K}(Z)$, the reduced $K$-theory of $Z$. Applying theorem 3.1 we obtain the following proposition.

Proposition 3.5. $K_{H}^{0}(Z) \cong \bigoplus_{i=1, i \neq n}^{2 n-1} H^{2 i}(Z ; \mathbb{Z}) \oplus \mathbb{Z}_{2 k} \cong H_{H}^{\text {even }}(Z)$.

The following proposition computes the odd twisted $K$-theory.

Proposition 3.6. $K_{H}^{1}(Z) \cong \bigoplus_{i=0}^{2 n-2} H^{2 i+1}(Z ; \mathbb{Z}) \oplus \mathbb{Z}_{2 l} \cong H_{H}^{o d d}(Z ; \mathbb{Z})$

Proof. We observe that since $E_{\infty}^{p, q}=0$ when $q$ is odd, we have that $\mathscr{F}^{0}=K_{H}^{\mathbf{1}}(Z)$ and $\mathscr{F}^{2 i}=\mathscr{F}^{2 i-1}$ for $i>0$. In particular, our filtration of $K_{H}^{\mathbf{1}}(Z)$ can be written as

$$
K_{H}^{\mathbf{1}}(Z) \supseteq \mathscr{F}^{1} \supseteq \mathscr{F}^{3} \supseteq \cdots \supseteq \mathscr{F}^{4 n-3} \supseteq \mathscr{F}^{4 n-1}=\{0\}
$$

where $\frac{K_{H}^{1}(Z)}{\mathscr{F}^{1}} \cong H^{1}(Z ; \mathbb{Z}), \frac{\mathscr{F}^{2 i-1}}{\mathscr{F}^{2 i+1}} \cong H^{2 i+1}(Z ; \mathbb{Z})$ for $1 \leq i \leq n-2$, and $\frac{\mathscr{F}^{4 n-3}}{\mathscr{F}^{4 n-1}} \cong \mathbb{Z}_{2 l}$. As $\mathscr{F}^{4 n-1}=\{0\}$, we immediately obtain $\mathscr{F}^{4 n-3}=\mathbb{Z}_{2 l}$.

The filtration determines short exact sequences

$$
0 \rightarrow \mathscr{F}^{2 i+1} \rightarrow \mathscr{F}^{2 i-1} \rightarrow \frac{\mathscr{F}^{2 i-1}}{\mathscr{F}^{2 i+1}} \rightarrow 0
$$

for each $0 \leq i \leq n-1$.

In the case that $i=n-1$, the short exact sequence tells us that $\mathscr{F}^{4 n-3}=\mathbb{Z}_{2 l}$, which we already knew.

In the case that $0 \leq i<n-1$, we note that the groups $H^{2 i+1}(Z ; \mathbb{Z})$, for $0 \leq i \leq n-1$, are torsion free. This implies that the above short exact sequences split for all $0 \leq i<n-1$.

These observations immediately imply

$$
K_{H}^{\mathbf{1}}(Z) \cong \bigoplus_{i=0}^{2 n-2} H^{2 i+1}(Z ; \mathbb{Z}) \oplus \mathbb{Z}_{2 l}
$$

which proves the first isomorphism in the statement of the proposition. The second isomorphism follows from the computations carried out in section 3.5.

The arguments we gave, via a spectral sequence, to compute $K_{H}^{*}(Z)$ can be applied to compute $K_{\widehat{H}}^{*}(\widehat{Z})$. As the details are exact analogous to what we did above for $Z$, we simply state the results in the following proposition. 
Proposition 3.7. We have the following isomorphisms

$$
\begin{aligned}
& K_{\widehat{H}}^{\mathbf{0}}(\widehat{Z}) \cong \bigoplus_{i=1, i \neq n}^{2 n-1} H^{2 i}(\widehat{Z} ; \mathbb{Z}) \oplus \mathbb{Z}_{2 l} \cong H_{\widehat{H}}^{\text {even }}(\widehat{Z} ; \mathbb{Z}) \\
& K_{\widehat{H}}^{\mathbf{1}}(\widehat{Z}) \cong \bigoplus_{i=0}^{2 n-2} H^{2 i}(\widehat{Z} ; \mathbb{Z}) \oplus \mathbb{Z}_{2 k} \cong H_{\widehat{H}}^{\text {odd }}(\widehat{Z} ; \mathbb{Z}) .
\end{aligned}
$$

From these computations we thus see that the spherical $T$-dual pairs $(Z, H)$ and $(\widehat{Z}, \widehat{H})$ have isomorphic twisted $K$-theories upto a degree shift.

Theorem 3.8. We have the following isomorphism

$$
K_{H}^{0 / 1}(Z) \cong K_{\widehat{H}}^{1 / 0}(\widehat{Z})
$$

\section{SPECTRAL SEQUENCES}

To show that the Chern character is a real isomorphism from the twisted $K$-theory of a closed manifold $X$ to the twisted cohomology of $X$, in [4] Atiyah and Segal consider two spectral sequences. The first of these is a generalisation of the Atiyah-Hirzebruch spectral sequence [4, Section 5], which computes the twisted $K$-theory of $X$, while the second computes the twisted cohomology of $X$. We give in this section the correct generalisations of these spectral sequences to the higher twisted setting (noting that the second of these has already been covered in great detail in [32]).

4.1. The higher twisted Atiyah-Hirzebruch spectral sequence. The Atiyah-Hirzebruch spectral sequence for $K$-theory was introduced for finite $\mathrm{CW}$ complexes $X$ (of which closed manifolds are examples) in the paper [3]. As pointed out in [4], there is an analogous spectral sequence for twisted $K$-theory.

Our goal in this subsection is to show that there is an Atiyah-Hirzebruch spectral sequence for higher twisted $K$-theory also, which generalises that in [4]. This spectral sequence will later be compared with that for higher twisted cohomology. To construct the Atiyah-Hirzebruch spectral sequence, we must shift our attention from manifolds to $C W$ complexes. Moreover, we will work with general twists, arising from classifying maps into $B A u t\left(\mathscr{O}_{\infty} \otimes \mathscr{K}\right)$, which need not correspond to cohomology classes as outlined in the Preliminaries.

Definition 4.1. By a pair with twist we mean a triple $(X, A, \lambda)$, where $X$ is a finite $C W$ complex, $A \subset X$ a $C W$ subcomplex, and $\lambda: X \rightarrow B A u t\left(\mathscr{O}_{\infty} \otimes \mathscr{K}\right)$ a twist over $X$. Let $\left(X, A, \lambda_{X}\right)$ and $\left(Y, B, \lambda_{Y}\right)$ be pairs with twist. A morphism $f:\left(X, A, \lambda_{X}\right) \rightarrow\left(Y, B, \lambda_{Y}\right)$ is a morphism $f$ of $C W$ pairs such that $\lambda_{Y} \circ f=\lambda_{X}$.

We remind the reader that, given a twist $\lambda: X \rightarrow B A u t\left(\mathscr{O}_{\infty} \otimes \mathscr{K}\right)$ and a closed subspace $A \subset X$, then denoting by $F_{\lambda}^{n}$ the associated bundle over $X$ with fibre $\Omega^{n}$ Fred $_{\mathscr{O}_{\infty} \otimes \mathscr{K}}$, the higher twisted $K$-theory group $K_{\lambda}^{n}(X, A)$ is the homotopy classes of sections of $F_{\lambda}^{n}$ that are equal to the identity outside of some compact set in $X-A$. Clearly now if $f:\left(X, A, \lambda_{X}\right) \rightarrow\left(Y, B, \lambda_{Y}\right)$ is a morphism of pairs with twist, then $f^{*} F_{\lambda_{Y}}^{n}=F_{\lambda_{X}}^{n}$, so that the pullback by $f$ of any section of $F_{\lambda_{Y}}^{n}$ is a section of $F_{\lambda_{X}}^{n}$. That is:

Proposition 4.2. The assignment $(X, A, \lambda) \mapsto K_{\lambda}^{n}(X, A)$ is a functor from the category of pairs with twist to the category of abelian groups. 
As identified by Pennig [41], one way of obtaining the six-term exact sequence in higher twisted $K$-theory is to use an identification with operator $K$-theory groups. For the purpose of showing that our Chern character is a real isomorphism, we choose here to take a different, more topological approach inspired by Atiyah [2].

Associated to the pair with twist $(X, A, \lambda)$, we have the sequence $(A, \emptyset, \lambda) \rightarrow(X, \emptyset, \lambda) \rightarrow(X, A, \lambda)$ of pairs with twist, which by functoriality induces the exact sequence

$$
K_{\lambda}^{n}(X, A) \stackrel{\text { ext }_{*}}{\longrightarrow} K_{\lambda}^{n}(X) \stackrel{\text { rest }_{*}}{\longrightarrow} K_{\lambda}^{n}(A)
$$

of $K$-theory groups. Here ext ${ }_{*}$ is induced by extension of sections by the identity, and rest ${ }_{*}$ is induced by restriction of sections. To obtain the corresponding long exact sequnece, we must give a connecting homomorphism $\partial: K_{\lambda}^{n}(A) \rightarrow K_{\lambda}^{n+1}(X, A)$. We follow the definition given by Atiyah for ordinary $K$-theory [2, p. 71-72], with some necessary modifications from Pennig [41] to deal with the fact that twists do not extend to one-point compactifications.

Following Pennig, define $c(X, A):=X \sqcup(A \times[0,1)) / \sim$, where $(a, 0) \in A \times[0,1)$ is glued to $a \in X$. We call this the open cone on $A$. The twist $\lambda$ over $X$ extends trivially to a twist $c \lambda$ over $c(X, A)$, and via a neighbourhood retract of $c(X, A)$ onto $X$ we have

$$
K_{\lambda}^{n+1}(X, A)=K_{c \lambda}^{n+1}(c(X, A)) .
$$

Associated then to the map $m:(c(X, A), \emptyset, c \lambda) \rightarrow(c(X, A), X, c \lambda)$ of pairs with twist, we obtain a map

$$
m^{*}: K_{c \lambda}^{n+1}(c(X, A), X) \rightarrow K_{c \lambda}^{n+1}(c(X, A))=K_{\lambda}^{n+1}(X, A) .
$$

Now, the space $c(X, A)-X$ is simply the product $A \times(0,1)$ (which we call the open suspension of $A)$. Thus if $\sigma$ is a section of $F_{c \lambda}^{n+1}$ over $c(X, A)-X$ which is equal to the identity outside of some compact set, we may define a new section $\tilde{\sigma}: A \rightarrow F_{\lambda}^{n+2}$ by the formula

$$
\tilde{\sigma}(a)\left(e^{2 \pi i t}\right):=\sigma(a, t), \quad a \in A, t \in S^{1}
$$

Note that well-definedness of $\tilde{\sigma}(a)$ as a function on the circle follows from the fact that $\sigma$ is equal to the identity outside of some compact subset of $A \times(0,1)$. The map $\sigma \mapsto \tilde{\sigma}$ descends to a well-defined map on homotopy classes, whose composite with the Bott periodicity isomorphism $K_{\lambda}^{n+2}(A) \cong K_{\lambda}^{n}(A)$ therefore defines a map

$$
\theta: K_{c \lambda}^{n+1}(c(X, A), X) \rightarrow K_{\lambda}^{n}(A) .
$$

We thus obtain our connecting homomorphism

$$
\partial_{n}:=m^{*} \circ \theta^{-1}: K_{\lambda}^{n}(A) \rightarrow K_{\lambda}^{n+1}(X, A) .
$$

Applying Bott periodicity and using similar arguments to those of Atiyah [2, Proposition 2.4.4], we therefore have the six-term sequence defined in topological terms.

Theorem 4.3. Let $(X, A, \lambda)$ be a pair with twist. Then associated to the sequence

$$
(A, \emptyset, \lambda) \rightarrow(X, \emptyset, \lambda) \rightarrow(X, A, \lambda)
$$

of pairs with twist we have the exact sequence 


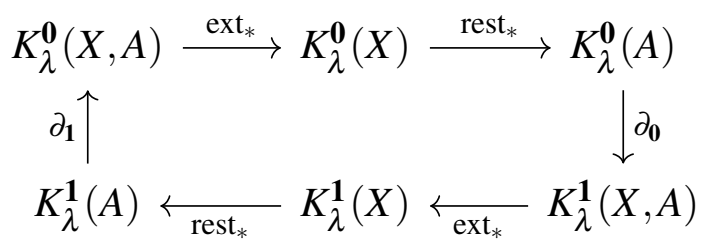

of twisted K-groups.

We use Theorem 4.3 to give an Atiyah-Hirzebruch spectral sequence computing $K_{\lambda}^{*}(X)$ as follows. Denote the $p$-skeleton of $X$ by $X^{p}$. Each inclusion $X^{p} \hookrightarrow X$ induces by a homomorphism $K_{\lambda}^{*}(X) \rightarrow$ $K_{\lambda}^{*}\left(X^{p}\right)$ on higher twisted $K$-theory induced by restricting sections of $F_{\lambda}$ to $X^{p}$. We thus filter the higher twisted $K$-theory groups $K_{\lambda}^{*}(X)$ of $X$ by the skeleta of $X$ :

$$
\mathscr{F}^{p} K_{\lambda}^{*}(X):=\operatorname{ker}\left(K_{\lambda}^{*}(X) \rightarrow K_{\lambda}^{*}\left(X^{p-1}\right)\right)=\operatorname{im}\left(K_{\lambda}^{*}\left(X, X^{p-1}\right) \rightarrow K_{\lambda}^{*}(X)\right), \quad p>0,
$$

taking in particular $\mathscr{F}^{0} K_{\lambda}^{*}(X)=K_{\lambda}^{*}(X)$. We have the following analogue of [3, Theorem 2.1].

Theorem 4.4 (Higher twisted Atiyah-Hirzebruch spectral sequence). Let $X$ be a finite $C W$ complex, equipped with a higher twist $P$. Then there is a spectral sequence $\left\{E_{r}^{p}(K)\right\}$ with

$$
E_{2}^{p}(K) \cong H^{p}(X, \mathbb{Z}), \quad E_{\infty}^{p}(K)=\bigoplus_{* \in \mathbb{Z}_{2}} \mathscr{F}^{p} K_{\lambda}^{p \bmod 2+*}(X) / \mathscr{F}^{p+1} K_{\lambda}^{p \bmod 2+*}(X),
$$

which computes $K_{\lambda}^{*}(X)$.

Proof. For each $p>0$, we have the sequence

$$
\left(X^{p-1}, \emptyset, \lambda\right) \rightarrow\left(X^{p}, \emptyset, \lambda\right) \rightarrow\left(X^{p}, X^{p-1}, \lambda\right)
$$

of pairs with twist, and thus by Theorem 4.3 we obtain the long exact sequence

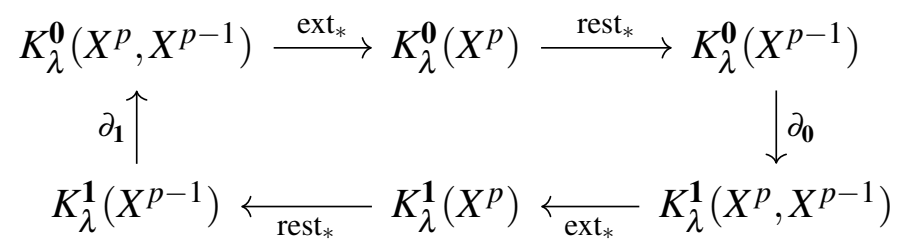

of higher twisted $K$-theory groups. We define $A_{1}^{p, *}(K):=K_{\lambda}^{*}\left(X^{p}\right), E_{1}^{p, *}(K):=K_{\lambda}^{*}\left(X^{p}, X^{p-1}\right)$, and denote the associated bigraded groups by

$$
A_{1}(K):=\bigoplus_{p \in \mathbb{Z}, * \in \mathbb{Z}_{2}} A_{1}^{p, *}(K), \quad E_{1}(K):=\bigoplus_{p \in \mathbb{Z}, * \in \mathbb{Z}_{2}} E_{1}^{p, *}(K) .
$$

From the maps in the exact sequence (4.2), we obtain:

(1) $\alpha_{1}(K): A_{1}(K) \rightarrow A_{1}(K)$ of bi-degree $(-1,0)$ induced by rest ${ }_{*}$,

(2) $\beta_{1}(K): A_{1}(K) \rightarrow E_{1}(K)$ of bi-degree $(0,0)$ induced by ext ${ }_{*}$, and

(3) $\gamma_{1}(K): E_{1}(K) \rightarrow A_{1}(K)$ of bi-degree $(1,1)$ induced by $\partial_{*}$,

and exactness of (4.2) therefore guarantees that

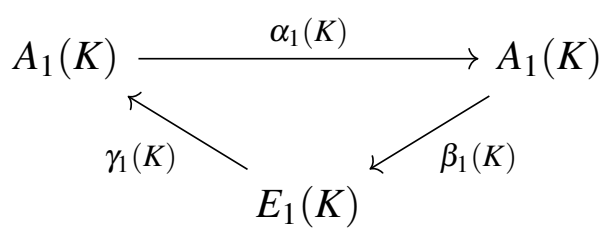


is an exact couple. The Atiyah-Hirzebruch spectral sequence $\left\{E_{r}^{p, *}(K)\right\}$ is now obtained from the derived couples of the exact couple (4.3), with differentials $d_{r}: E_{r}^{p, *}(K) \rightarrow E_{r}^{p+r, *+1}(K)$ of bidegree $(r, 1)$. Due to the fact that $X^{p}=X$ for $p$ sufficiently large, this spectral sequence stabilises with $E_{\infty}(K)$ term

$$
E_{\infty}^{p, *}(K)=\mathscr{F}^{p} K_{\lambda}^{p \bmod 2+*}(X) / \mathscr{F}^{p+1} K_{\lambda}^{p \bmod 2+*}(X),
$$

hence converges to $K_{\lambda}^{*}(X)$ as claimed.

Finally let us show that the $E_{2}(K)$ term is as claimed. We have that

$$
E_{1}^{p, *}(K)=K_{\lambda}^{*}\left(X^{p}, X^{p-1}\right)
$$

is the homotopy classes of sections of the $\Omega^{*}$ Fred $_{\mathscr{O}_{\infty} \otimes \mathscr{K}}$-bundle $F_{\lambda}^{*}$ over $X^{p}-X^{p-1}$ which are the identity outside some compact set. Now $X^{p}-X^{p-1}$ consists precisely of the interiors of the $p$-cells of $X$, and since the interior of any $p$-cell is contractible $\left.F_{\lambda}^{*}\right|_{X^{p}-X^{p-1}}$ is trivialisable. Therefore $K_{\lambda}^{*}\left(X^{p}, X^{p-1}\right)$ coincides with the ordinary $K$-theory $K^{*}\left(X^{p}, X^{p-1}\right)$ of the pair $\left(X^{p}, X^{p-1}\right)$, and the ordinary relative Chern character restricts thereon to an isomorphism $K^{\mathbf{0}}\left(X^{p}, X^{p-1}\right) \cong$ $H^{\text {even }}\left(X^{p}, X^{p-1} ; \mathbb{Z}\right)$ with relative singular cohomology with coefficients in $\mathbb{Z}$. Naturality of the Chern character as a transformation of (generalised) cohomology functors then says that $d_{1}(K)$ is the cellular coboundary, giving the result.

4.2. Singular de Rham theory. To show that the Chern character for higher twisted $K$-theory is a real isomorphism, we must consider twisted cohomology for CW complexes. Since the Chern character form we constructed earlier on the Fred $_{\mathscr{O}_{\infty} \otimes \mathscr{K}}$-bundle $Y_{2 k+1}$ over $S^{2 k+1}$ is a differential form, in constructing the Chern character for a CW complex with twist $(X, \lambda)$, we must use a cohomology theory which is simultaneously compatible with $\mathrm{CW}$ complexes and with differential forms. As pointed out by Atiyah-Segal [4], singular de Rham theory is perfect for this role. We review this theory for the reader's convenience here in the convenient category of diffeological spaces, whose definition we now recall following [30, Definition 1.5].

Definition 4.5. Let $X$ be a set. A parametrisation is a function $\varphi: U \rightarrow X$, where $U$ is some open subset in some finite-dimensional Euclidean space. A diffeology on $X$ is a set D of parametrisations for which the following hold.

(1) Every constant parametrisation is contained in $D$.

(2) If $\varphi: U \rightarrow X$ is a parametrisation such that every $u \in U$ admits an open neighbourhood $V \subset U$ for which $\left.\varphi\right|_{V}: V \rightarrow X$ is an element of $D$, then $\varphi$ is an element of $D$.

(3) If $\varphi: U \rightarrow X$ is an element of $D, V$ is any open subset in some finite-dimensional Euclidean space and $f: V \rightarrow U$ is a smooth function, then $\varphi \circ f: V \rightarrow X$ is contained in $D$.

A set with a diffeology is called a diffeological space, and the elements of the diffeology are called plots. A function $f: X \rightarrow Y$ of diffeological spaces is said to be smooth if, for every plot $\varphi: U \rightarrow X$ of $X$, the composite $f \circ \varphi: U \rightarrow Y$ is a plot of $Y$. Any subset $S$ of a diffeological space $X$ can be equipped with the subspace diffeology, for which a parametrisation $\varphi: U \rightarrow S$ is a plot if and only if its composition with the inclusion $S \hookrightarrow X$ is a plot of $X$.

Diffeological spaces were originally introduced by Soriau [46], who was motivated by problems in quantisation. They are vast generalisations of manifolds, and are used to apply tools of differential geometry to spaces that are not well-behaved by traditional standards, for instance orbifolds [29] 
and, increasingly, leaf spaces of foliations [25, 26, 34, 35]. Here we choose to work in the diffeological category out of convenience - it allows us to use a single language to speak of the singular de Rham theory of both topological spaces and manifolds as we will now describe.

Any topological space can be equipped with a diffeology, called its continuous diffeology [19, Section 3], whose plots are all those parametrisations which are continuous. Smooth maps between topological spaces $X$ and $Y$ with the continuous diffeology are precisely the continuous maps $X \rightarrow$ $Y$. Moreover, any manifold has a standard diffeology whose plots are all those parametrisations which are smooth in the manifold sense, and a diffeologically smooth map of manifolds is the same thing as a smooth map in the usual sense. In particular, every geometric simplex

$$
\Delta^{n}:=\left\{\left(x_{1}, \ldots, x_{n}\right) \in \mathbb{R}^{n}: 0 \leq x_{1} \leq \cdots \leq x_{n} \leq 1\right\}
$$

is a diffeological subspace of Euclidean space. The singular de Rham complex of a diffeological space is defined as follows.

Definition 4.6. Let $X$ be a diffeological space, and let $k \geq 0$. A singular simplex is a diffeologically smooth map $\sigma: \Delta^{l} \rightarrow X$. A singular $k$-form $\omega$ on $X$ is a map which assigns to each singular simplex $\sigma: \Delta^{l} \rightarrow X$ a differential $k$-form $\omega(\sigma) \in \Omega^{k}\left(\Delta^{l}\right)$, subject to the constraint that the restriction of $\omega(\sigma)$ to the $i^{\text {th }}$ face of the geometric simplex $\Delta^{l}$ is equal to $\omega\left(\varepsilon_{i}(\sigma)\right)$, where $\varepsilon_{i}$ is the $i^{\text {th }}$ face map. The set $\Omega_{\text {sing }}^{k}(X)$ of all singular $k$-forms is a real vector space under componentwise operations:

$$
(\alpha \omega+\beta \kappa)(\sigma):=\alpha \omega(\sigma)+\beta \kappa(\sigma), \quad \alpha, \beta \in \mathbb{R}, \omega, \kappa \in \Omega_{\text {sing }}^{k}(X) .
$$

The direct sum $\Omega_{\text {sing }}^{*}(X):=\bigoplus_{k \geq 0} \Omega_{\text {sing }}^{k}(X)$ is a differential graded algebra under pointwise operations:

$$
(d \omega)(\sigma):=d \omega(\sigma), \quad(\omega \wedge \kappa)(\sigma):=\omega(\sigma) \wedge \kappa(\sigma), \quad \omega, \kappa \in \Omega^{*}(X) .
$$

The differential graded algebra $\Omega_{\text {sing }}^{*}(X)$ is called the singular de Rham complex of $X$, and its cohomology denoted $H_{\text {sing-dR }}^{*}(X)$.

For manifolds, singular de Rham cohomology is related to ordinary de Rham cohomology by a pullback map. For a proof we refer the reader to [31, Theorem 2.4].

Theorem 4.7. Let $X$ be a manifold. Then the map $\alpha: \Omega^{*}(X) \rightarrow \Omega_{\text {sing }}^{*}(X)$ defined by

$$
\alpha(\omega)(\sigma):=\sigma^{*} \omega
$$

is a quasi-isomorphism.

There is a diffeological de Rham theorem [31, Corollary 2.5], which relates singular de Rham theory to singular theory.

Theorem 4.8. Let $X$ be a diffeological space, and denote by $C^{*}(X)$ the complex of smooth singular cochains of $X$. Then the integration map $\int: \Omega_{\text {sing }}^{*}(X) \rightarrow C^{*}(X)$ defined by

$$
\left(\int \omega\right)\left(\sigma: \Delta^{l} \rightarrow X\right):=\int_{\Delta^{l}} \omega(\sigma), \quad \omega \in \Omega_{\text {sing }}^{*}(X)
$$

is a cochain map, and is moreover a quasi-isomorphism, inducing an isomorphism $H_{\text {sing-dR }}^{*}(X) \cong$ $H^{*}(X ; \mathbb{R})$ on cohomology. 
In particular, the singular de Rham cohomology of a topological space is isomorphic to the ordinary singular cohomology with real coefficients. The singular de Rham complexes of diffeological spaces are functorial under smooth maps.

Proposition 4.9. Let $f: X \rightarrow Y$ be a smooth map of diffeological spaces. Then the formula

$$
\left(f^{*} \omega\right)(\sigma):=\omega(f \circ \sigma), \quad \omega \in \Omega_{\text {sing }}^{*}(Y)
$$

defines a map $f^{*}: \Omega_{\text {sing }}^{*}(Y) \rightarrow \Omega_{\text {sing }}^{*}(X)$ of cochain complexes. In particular, if $X$ and $Y$ are manifolds, then the diagram

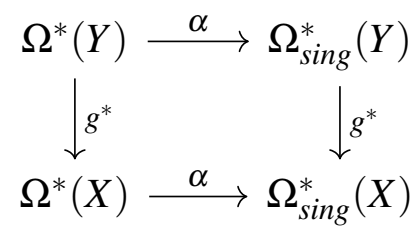

commutes.

Proof. The first part is clear. For the second, simply fix $\omega \in \Omega^{*}(Y)$ and observe that

$$
g^{*}(\alpha(\omega))(\sigma)=\alpha(\omega)(g \circ \sigma)=(g \circ \sigma)^{*} \omega=\sigma^{*}\left(g^{*} \omega\right)=\alpha\left(g^{*} \omega\right)(\sigma)
$$

for any smooth singular simplex $\sigma$ of $X$.

We have the following crucial lemma, which relies on the extendability property [24, Lemma 9.4] of the differential forms on geometric simplices.

Lemma 4.10. Let $f: X \rightarrow Y$ be an injective smooth map of diffeological spaces. Then $f^{*}$ : $\Omega_{\text {sing }}^{*}(Y) \rightarrow \Omega_{\text {sing }}^{*}(X)$ is surjective.

Proof. Fix $\omega \in \Omega_{\text {sing }}^{*}(X)$. We must construct a singular form $\tilde{\omega} \in \Omega_{\text {sing }}^{*}(Y)$ such that $f^{*} \tilde{\omega}=\omega$. Let $\tilde{\sigma}: \Delta^{n} \rightarrow Y$ be a smooth singular simplex. If $\tilde{\sigma}=f \circ \sigma$ for some singular simplex $\sigma$ of $X$, then by injectivity of $f, \sigma$ is uniquely characterised by this property and we simply define

$$
\tilde{\omega}(\tilde{\sigma}):=\omega(f \circ \sigma) \text {. }
$$

In general, while $\tilde{\sigma}$ may not itself be of the form $f \circ \sigma$, it may have faces which are of this form. To take care of this subtlety we must in general construct $\tilde{\omega}$ inductively.

Let $v_{0}, \ldots, v_{n}$ be the vertices of $\Delta^{n}$. We define

$$
\tilde{\omega}\left(\left[v_{i}\right]\right):= \begin{cases}\omega\left(\sigma_{i}\right) & \text { if }\left.\tilde{\sigma}\right|_{v_{i}}=f \circ \sigma_{i} \text { for some } 0 \text {-simplex } \sigma_{i} \text { in } X \\ 0 & \text { otherwise. }\end{cases}
$$

Given a multi-index $I_{k}:=\left(i_{1}, \ldots, i_{k}\right)$, denote by $\left[v_{I_{k}}\right]$ the corresponding $(k-1)$-face

$$
\left[v_{I_{k}}\right]:=\left[v_{i_{1}}, \ldots v_{i_{k}}\right] \text {, }
$$

and for $1 \leq j \leq k$ denote by $\left[\varepsilon_{j} v_{I_{k}}\right]$ the $j^{t h}$ face of $\left[v_{I_{k}}\right]$. Suppose inductively that we have forms $\tilde{\omega}\left(\left[v_{I_{k}}\right]\right)$ defined on all the $(k-1)$-faces of $\tilde{\sigma}$ which agree on intersections, and given any $k$-face $\left[v_{I_{k+1}}\right]$ of $\tilde{\sigma}$ denote by

$$
\operatorname{ext}\left(\tilde{\omega}\left(\left[\varepsilon_{1} v_{I_{k+1}}\right]\right), \ldots, \tilde{\omega}\left(\left[\varepsilon_{k+1} v_{I_{k+1}}\right]\right)\right)
$$


the form on $v_{I_{k+1}}$ extending the $\tilde{\omega}_{\varepsilon_{i} v_{I_{k+1}}}$ obtained via [24, Lemma 9.4]. Then we define

$$
\tilde{\omega}\left(\left[v_{I_{k+1}}\right]\right):= \begin{cases}\omega\left(\sigma_{I_{k+1}}\right) & \text { if }\left.\tilde{\sigma}\right|_{\left[v_{I_{k+1}}\right]}=f \circ \sigma_{I_{k+1}}, \text { for } \sigma_{I_{k+1}}: \Delta^{k} \rightarrow X \\ \operatorname{ext}\left(\tilde{\omega}\left(\left[\varepsilon_{1} v_{I_{k+1}}\right]\right), \ldots, \tilde{\omega}\left(\left[\varepsilon_{k+1} v_{\left.I_{k+1}\right]}\right]\right)\right. & \text { otherwise }\end{cases}
$$

to obtain a family of forms $\tilde{\omega}\left(\left[v_{I_{k+1}}\right]\right)$ associated to the $k$-faces of $\tilde{\sigma}$. After $n$ steps, we obtain the desired form $\tilde{\omega}(\tilde{\sigma}) \in \Omega^{*}\left(\Delta^{n}\right)$, and if in particular $\tilde{\sigma}=f \circ \sigma$ for some simplex $\sigma: \Delta^{n} \rightarrow X$, then $\tilde{\omega}(\tilde{\sigma})=\omega(\sigma)$ by construction.

Doing this construction for each singular simplex $\tilde{\sigma}$ of $Y$, we obtain a singular form $\tilde{\omega} \in \Omega_{\text {sing }}^{*}(Y)$, and if $\sigma: \Delta^{n} \rightarrow X$ is any singular simplex then

$$
\left(f^{*} \tilde{\omega}\right)(\sigma)=\tilde{\omega}(f \circ \sigma)=\omega(\sigma) .
$$

Thus $f^{*}$ is surjective as claimed.

Theorem 4.8 implies a similar isomorphism for relative cohomology groups, which we now define.

Definition 4.11. Let $X$ be a diffeological space, and let $A \subset X$ be a diffeological subspace. We denote by $\Omega_{\text {sing }}^{*}(X, A)$ the differential graded subalgebra of $\Omega_{\text {sing }}^{*}(X)$ consisting of singular forms $\omega$ such that $\omega(\sigma)=0$ whenever $\sigma: \Delta^{l} \rightarrow A$ is a singular simplex of $A$. The cohomology of $\Omega_{\text {sing }}^{*}(X, A)$ is denoted $H_{\text {sing-dR }}^{*}(X, A)$.

If $X$ is any diffeological space and $A$ any diffeological subspace, then the restriction map from $\Omega_{\text {sing }}^{*}(X)$ to $\Omega_{\text {sing }}^{*}(A)$ is surjective by Lemma 4.10, and lifts of singular forms in $A$ to singular forms in $X$ are unique up to elements of $\Omega_{\text {sing }}^{*}(X, A)$. Thus $\Omega_{\text {sing }}^{*}(A)$ is isomorphic to the quotient $\Omega_{\text {sing }}^{*}(X) / \Omega_{\text {sing }}^{*}(X, A)$. Therefore we have an exact sequence

$$
0 \rightarrow \Omega_{\text {sing }}^{*}(X, A) \stackrel{\imath}{\rightarrow} \Omega_{\text {sing }}^{*}(X) \stackrel{\pi}{\rightarrow} \Omega_{\text {sing }}^{*}(A) \rightarrow 0
$$

of cochain complexes, where $l$ is the inclusion and where $\pi$ is restriction.

Now the integration map described in Theorem 4.8 sends $\Omega_{\text {sing }}^{*}(X, A)$ into the relative singular cochains $C^{*}(X, A ; \mathbb{R})$ - that is, cochains which vanish on singular simplices which map into $A$. We then have the commuting diagram

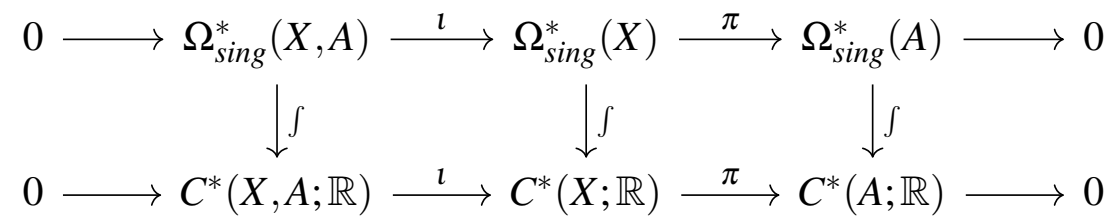

of cochain complexes. Passing to the long exact sequences guaranteed by the zig zag lemma, we obtain commuting diagrams

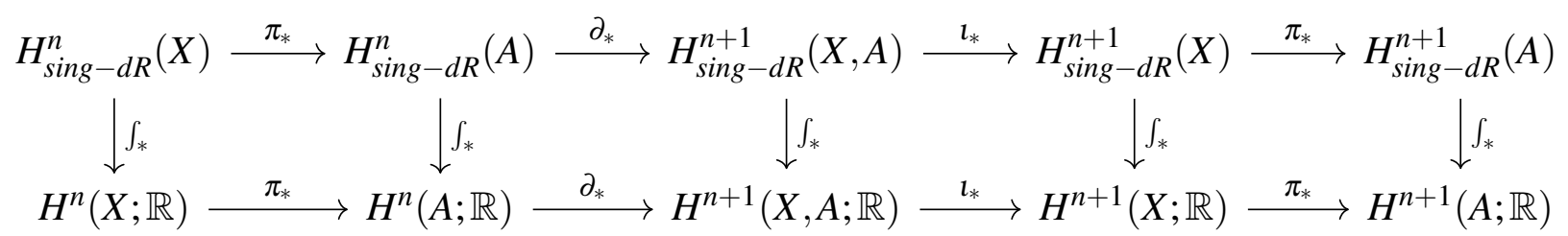

for each $n$, with exact rows. The five lemma together with Theorem 4.8 therefore proves the following. 
Proposition 4.12. For a diffeological space $X$ and any subspace $A$ of $X$, the integration map from singular de Rham forms to singular cochains induces an isomorphism $H_{\text {sing-dR }}^{*}(X, A) \cong$ $H^{*}(X, A ; \mathbb{R})$.

We conclude this subsection by considering the relationship between the singular de Rham theories of a manifold when equipped with its standard manifold diffeology versus when it is equipped with its continuous diffeology.

Proposition 4.13. Let $X$ be a manifold, and denote by $X^{t}$ and $X^{m}$ the set $X$ regarded with its continuous diffeology and with its manifold diffeology respectively. The identity map id: $X^{m} \rightarrow X^{t}$ is smooth, and the induced map $\mathrm{id}^{*}: \Omega_{\text {sing }}^{*}\left(X^{t}\right) \rightarrow \Omega_{\text {sing }}^{*}\left(X^{m}\right)$ is a quasi-isomorphism. Consequently, every closed element of $\Omega^{*}\left(X^{m}\right)$ has a lift to a closed element of $\Omega_{\text {sing }}^{*}\left(X^{t}\right)$.

Proof. The first claim follows from commutativity of the diagram

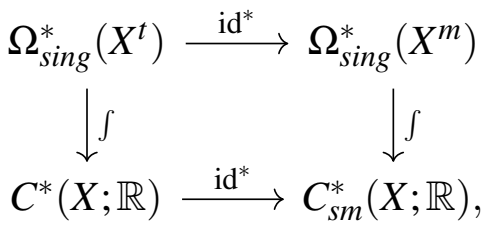

where $C_{s m}^{*}(X ; \mathbb{R})$ denotes the cochain complex of singular cochains on smooth singular simplices, and where the bottom id ${ }^{*}$ denotes restriction of cochains from continuous to smooth. Since the bottom $\mathrm{id}^{*}$ is a quasi-isomorphism [49, p. 205] and since both integration maps are quasiisomorphisms by Theorem 4.8, the top id* is also a quasi-isomorphism. The second claim then follows from injectivity of id together with Lemma 4.10. Indeed, if $\omega \in \Omega_{\operatorname{sing}}^{n}\left(X^{m}\right)$ is closed, then since $\mathrm{id}^{*}$ is a surjective quasi-isomorphism we can find closed $\kappa_{1} \in \Omega_{\text {sing }}^{n}\left(X^{t}\right)$ and $\kappa_{2} \in \Omega_{\text {sing }}^{n-1}\left(X^{t}\right)$ such that

$$
\mathrm{id}^{*}\left(\kappa_{1}\right)=\omega+d\left(\mathrm{id}^{*} \kappa_{2}\right) .
$$

Then $\tilde{\omega}:=\kappa_{1}-d \kappa_{2}$ is a closed lift of $\omega$.

4.3. The higher twisted cohomology spectral sequence. In contrast to the twisted cohomology spectral sequences considered in [4] and [32], our twisted cohomology spectral sequence will be constructed in a very similar fashion to the Atiyah-Hirzebruch spectral sequence, in terms of filtrations by skeleta. Let us fix for the entirety of this subsection a finite CW complex $X$, with a cohomological twist $[H] \in H^{2 k+1}(X ; \mathbb{Z})$ that will be represented by a singular $(2 k+1)$-form $H \in \Omega_{\text {sing }}^{2 k+1}(X)$.

As pointed out by Atiyah and Segal, since $H$ is an odd-degree singular form, the twisted differential

$$
D:=d-H \wedge
$$

squares to zero on the singular de Rham complex $\Omega_{\text {sing }}^{*}(X)$ of $X$, and preserves the $\mathbb{Z}_{2}$-grading thereon. We can therefore consider the $\mathbb{Z}_{2}$-graded twisted singular de Rham cohomology $H_{H}^{*}(X)$ of the complex $\left(\Omega_{\text {sing }}^{*}(X), D\right)$. Similar remarks hold for the relative groups $(X, A)$ for any $\mathrm{CW}$ subcomplex $A$ of $X$, and we may consider the twisted relative groups $H_{H}^{*}(X, A)$. The following theorem now follows from the zig zag lemma. 
Theorem 4.14. Let $(X, A, H)$ be a $C W$ pair with cohomological twist represented by a singular $(2 k+1)$-form $H$. Associated to the sequence $(A, \emptyset, H) \rightarrow(X, \emptyset, H) \rightarrow(X, A, H)$ of pairs with twist, we have the short exact sequnece

$$
0 \rightarrow \Omega_{\text {sing }}^{*}(X, A) \stackrel{\imath}{\rightarrow} \Omega_{\text {sing }}^{*}(X) \stackrel{\pi}{\rightarrow} \Omega_{\text {sing }}^{*}(A) \rightarrow 0
$$

of (twisted) differential graded algebras, giving rise to a six term exact sequence

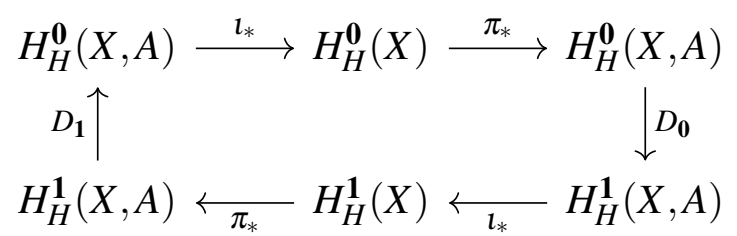

of twisted cohomology groups.

Note that the connecting homomorphism in the six term sequence of Theorem 4.14 is induced by the twisted differential $D$ after identifying $\Omega_{\text {sing }}^{*}(A)$ with $\Omega_{\text {sing }}^{*}(X) / \Omega_{\text {sing }}^{*}(X, A)$ as in the previous subsection.

Consider now the filtration of $X$ by its skeleta $X^{p}$. Exactly as for twisted $K$-theory, we can consider the corresponding filtration

$$
\mathscr{F}^{p} H_{H}^{*}(X):=\operatorname{ker}\left(H_{H}^{*}(X) \rightarrow H_{H}^{*}\left(X^{p-1}\right)\right)=\operatorname{im}\left(H_{H}^{*}\left(X, X^{p-1}\right) \rightarrow H_{H}^{*}(X)\right)
$$

of twisted cohomology by the skeleta of $X$. Now substituting $K^{*}$ for $H^{*}$, and ext ${ }_{*}$, rest ${ }_{*}$ and $\partial_{*}$ for $\imath_{*}, \pi_{*}$ and $D_{*}$ in Theorem 4.4, we have the following theorem giving a spectral sequence which computes $H_{H}^{*}(X)$.

Theorem 4.15. Let $(X, H)$ be a finite $C W$ complex with twist represented by $H \in \Omega_{\text {sing }}^{2 k+1}(X)$. Then there is a spectral sequence $\left\{E_{r}^{p}(H)\right\}$ with

$$
E_{2}^{p}(H) \cong H^{p}(X, \mathbb{Z}), \quad E_{\infty}^{p}(H)=\bigoplus_{* \in \mathbb{Z}_{2}} \mathscr{F}^{p} H_{H}^{p \bmod 2+*}(X) / \mathscr{F}^{p+1} H_{H}^{p \bmod 2+*}(X),
$$

which computes $H_{H}^{*}(X)$.

Proof. As mentioned, the proof of Theorem 4.4 applies almost without change. Let us just be careful about specifying the $E_{1}$ page. The $E_{1}$ page of this spectral sequence comes to us from an exact couple built out of the six term sequence of Theorem 4.14 with $X$ replaced by $X^{p}$ and $A$ replaced by $X^{p-1}$. Thus

$$
E_{1}^{p, *}(H)=H_{H}^{*}\left(X^{p}, X^{p-1}\right)
$$

Now the action of the twisted differential on $\Omega^{*}\left(X^{p}, X^{p-1}\right)$ is determined by the restriction of $H$ to $X^{p}-X^{p-1}$, which is a disjoint union of contractible open $p$-balls. Therefore $H$ is exact on $X^{p}-$ $X^{p-1}$, and we may choose a transgression of $H$ so as to obtain an identification of $H_{H}^{*}\left(X^{p}, X^{p-1}\right)$ with the relative singular de Rham cohomology $H_{\text {sing-dR }}^{*}\left(X^{p}, X^{p-1}\right.$ ) (see [4, Section 6, Remark (ii)]), and hence with the ordinary singular cohomology $H^{*}\left(X^{p}, X^{p-1} ; \mathbb{R}\right)$ by the integration map of Theorem 4.8. That the $E_{2}$ page is as claimed will follow from our comparison of the spectral sequences $E_{r}(K)$ and $E_{r}(H)$ via the Chern character in the next subsection (see Theorem 4.19), so we do not carry out the computation here. 
4.4. Comparison of the spectral sequences. Recall from Section 2 that even and odd twisted Chern character forms $\mathrm{Ch}_{2 k+1}^{\mathbf{0}}$ and $\mathrm{Ch}_{2 k+1}^{\mathbf{1}}$ have been constructed on the bundles $Y_{2 k+1}$ and $L_{2 k+1}$ over $S^{2 k+1}$, with fibres Fred $_{\mathscr{O}_{\infty} \otimes \mathscr{K}}$ and $\Omega$ Fred $\mathscr{O}_{\infty} \otimes \mathscr{K}$ respectively. We require the following.

Lemma 4.16. The pullback $\operatorname{id}_{Y_{2 k+1}}^{*}\left(C h_{2 k+1}^{\mathbf{0}}\right)$ of $C h_{2 k+1}^{\mathbf{0}} \in \Omega^{*}\left(Y_{2 k+1}\right)$ by the identity section $\operatorname{id}_{Y_{2 k+1}}$ : $S^{2 k+1} \rightarrow Y_{2 k+1}$ is equal to zero. Similarly, the pullback $\operatorname{id}_{L_{2 k+1}}^{*}\left(C h_{2 k+1}^{\mathbf{1}}\right)$ of $C h_{2 k+1}^{\mathbf{1}} \in \Omega^{*}\left(L_{2 k+1}\right)$ by the identity section $\mathrm{id}_{L_{2 k+1}}: S^{2 k+1} \rightarrow L_{2 k+1}$ is zero.

Proof. We prove the result only for $C h_{2 k+1}^{\mathbf{0}}$, as the odd case is similar. About any point in $S^{2 k+1}$, we can find an open neighbourhood $U$ and a trivialisation $\left.Y_{2 k+1}\right|_{U} \cong U \times$ Fred $_{\mathscr{O}_{\infty} \otimes \mathscr{K}}$ with respect to which $C h_{2 k+1}^{\mathbf{0}}$ is just the pullback of a Chern character form on $F r e d_{\mathscr{O}_{\infty} \otimes \mathscr{K}}$ by the projection $\pi_{2}: U \times$ Fred $_{\mathscr{O}_{\infty} \otimes \mathscr{K}} \rightarrow$ Fred $_{\mathscr{O}_{\infty} \otimes \mathscr{K}}$ onto the second factor. We then have that $\left.\pi_{2} \circ \mathrm{id}_{Y_{2 k+1}}\right|_{U}$ is the constant map $U \rightarrow\left\{\operatorname{id}_{\text {Fred }_{\mathscr{\infty} \infty} \otimes \mathscr{K}}\right\}$, giving the result.

By a finite $C W$ pair with cohomotopy twist we mean a triple $(X, A, H)$, where $X$ is a finite $C W$ complex, $A$ a CW subcomplex, and $H=\tilde{H}^{*}(\overline{\alpha(\eta)}) \in \Omega_{\text {sing }}^{2 k+1}(X)$ a singular de Rham form associated to a cohomotopy representative $\tilde{H}: X \rightarrow S^{2 k+1}$. Here $\eta \in \Omega^{2 k+1}\left(S^{2 k+1}\right)$ is the form used to construct $C h_{2 k+1}^{\mathbf{0}}$ and $C h_{2 k+1}^{\mathbf{1}}$ as in Section 2, and $\overline{\alpha(\eta)}$ is an extension of $\eta$ to a continuous singular de Rham form as in Theorem 4.7 and Proposition 4.13.

As in Section 2, we think of $K_{\lambda}^{\mathbf{0}}(X)$ as homotopy classes of lifts of the classifying map $\lambda: X \rightarrow$ $S^{2 k+1}$ to a map $F: X \rightarrow Y_{2 k+1}$. Similarly, $K_{\lambda}^{\mathbf{1}}(X)$ can be described as homotopy classes $G: X \rightarrow$ $L_{2 k+1}$ of $\lambda$. The relative twisted Chern character for CW pairs with cohomotopy twist may now be defined as follows.

Definition 4.17. Let $(X, A, H)$ be a finite $C W$ pair with cohomotopy twist. Let $\alpha$ denote the map from Theorem 4.7 sending manifold de Rham forms to singular de Rham forms. The even twisted Chern character is the map $C h_{H}^{\mathbf{0}}: K_{[H]}^{\mathbf{0}}(X, A) \rightarrow H_{H}^{\mathbf{0}}(X, A)$ defined by

$$
C h_{H}^{\mathbf{0}}([F]):=\left[F^{*} \overline{\alpha\left(C h_{2 k+1}^{\mathbf{0}}\right)}\right],
$$

where $F: X \rightarrow Y_{2 k+1}$ is a lift of $\tilde{H}: X \rightarrow S^{2 k+1}$ which coincides with the identity section outside of some compact set in $X-A$, and where $\overline{\alpha\left(C h_{2 k+1}^{\mathbf{0}}\right)}$ is any extension of $\alpha\left(C h_{2 k+1}^{\mathbf{0}}\right)$ to a closed singular form for $Y_{2 k+1}$ with its continuous diffeology as in Proposition 4.13. Similarly, the odd twisted Chern character is the map $C h_{H}^{1}: K_{[H]}^{\mathbf{1}}(X, A) \rightarrow H_{H}^{\mathbf{1}}(X, A)$ defined by

$$
C h_{H}^{\mathbf{1}}([G]):=\left[G^{*} \overline{\alpha\left(C h_{2 k+1}^{\mathbf{1}}\right)}\right],
$$

where $G: X \rightarrow L_{2 k+1}$ is a lift of $\tilde{H}: X \rightarrow S^{2 k+1}$ which coincides with the identity section outside of some compact set in $X-A$, and where $\overline{\alpha\left(C h_{2 k+1}^{\mathbf{1}}\right)}$ is any extension of $\alpha\left(C h_{2 k+1}^{\mathbf{1}}\right)$ to a closed singular form for $L_{2 k+1}$ with its continuous diffeology as in Proposition 4.13.

Let us remark that the odd and even twisted Chern characters do indeed take values in the claimed relative cohomology groups. Indeed, if $\sigma: \Delta^{l} \rightarrow A$ is any singular simplex and $F: X \rightarrow Y_{2 k+1}$ is any lift of $\tilde{H}$ which coincides with the identity outside of some compact set in $X-A$, then $F \circ \sigma=\operatorname{id}_{Y_{2 k+1}} \circ \tilde{H} \circ \sigma$. Since $\operatorname{id}_{Y_{2 k+1}}^{*} C h_{2 k+1}^{0}$ is the zero form by Lemma 4.16, for any singular 
simplex $\sigma$ in $A$ we apply Proposition 4.9 to see that

$$
\begin{aligned}
\left(F^{*} \overline{\alpha\left(C h_{2 k+1}^{\mathbf{0}}\right)}\right)(\sigma) & =\overline{\alpha\left(C h_{2 k+1}^{\mathbf{0}}\right)}\left(\mathrm{id}_{Y_{2 k+1}} \circ \tilde{H} \circ \sigma\right)=\left(\operatorname{id}_{Y_{2 k+1}}^{*} \overline{\alpha\left(C h_{2 k+1}^{\mathbf{0}}\right)}\right)(\tilde{H} \circ \sigma) \\
& =\overline{\left(\operatorname{id}_{Y_{2 k+1}}^{*} \alpha\left(C h_{2 k+1}^{\mathbf{0}}\right)\right)}(\tilde{H} \circ \sigma)=\overline{\alpha\left(\operatorname{id}_{Y_{2 k+1}^{*}}^{*} C h_{2 k+1}^{\mathbf{0}}\right)}(\tilde{H} \circ \sigma)=0,
\end{aligned}
$$

so that $F^{*} C h_{2 k+1}^{\mathbf{0}} \in \Omega_{\text {sing }}^{*}(X, A)$. Here the third equality follows from the fact that $\operatorname{id}_{Y_{2 k+1}}^{*} \overline{\alpha\left(C h_{2 k+1}^{\mathbf{0}}\right)}$ is an extension of $\mathrm{id}_{Y_{2 k+1}}^{*} \alpha\left(C h_{2 k+1}^{\mathbf{0}}\right)$. Similar arguments apply for $C h_{H}^{\mathbf{1}}$. The Chern character can now be seen to be a natural transformation of generalised cohomology theories.

Theorem 4.18. The twisted Chern character is a natural transformation of generalised cohomology theories from the category of finite $C W$ pairs with cohomotopy twist to the category of abelian groups. That is, given a finite CW pair with cohomotopy twist $(X, A, H)$, for each $*=\mathbf{0}, \mathbf{1}$ the diagram

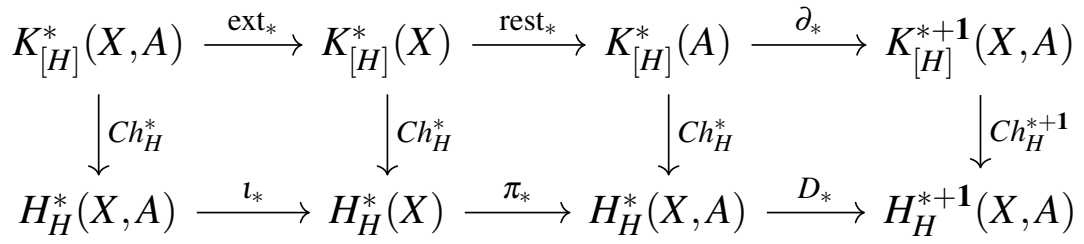

commutes.

Proof. To show commutativity of the first two squares, it suffices to show that if $f:\left(X, A, H_{X}\right) \rightarrow$ $\left(Y, B, H_{Y}\right)$ is a morphism of finite $\mathrm{CW}$ pairs with cohomotopy twist, so that $\tilde{H}_{Y} \circ f=\tilde{H}_{X}$, then the square

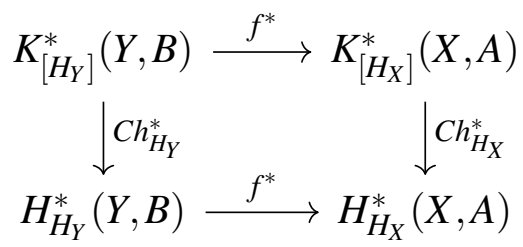

commutes. Suppose that $*=\mathbf{0}$. The $*=\mathbf{1}$ case follows by essentially the same argument. Then given a lift $F: Y \rightarrow Y_{2 k+1}$ of $\tilde{H}_{Y}$ which coincides with the identity section outside of some compact subset of $Y-B$, we compute

$$
\begin{aligned}
C h^{\mathbf{0}}\left(f^{*}[F]\right) & =C h^{\mathbf{0}}([F \circ f])=\left[(F \circ f)^{*} \overline{\alpha\left(C h_{2 k+1}^{\mathbf{0}}\right)}\right]=\left[f^{*}\left(F^{*} \overline{\alpha\left(C h_{2 k+1}^{\mathbf{0}}\right)}\right)\right] \\
& =f^{*}\left[F^{*} \overline{\alpha\left(C h_{2 k+1}^{\mathbf{0}}\right)}\right]=f^{*} C h^{\mathbf{0}}([F])
\end{aligned}
$$

giving the claimed commutativity. Commutativity of the final square follows from a retraction of the open suspension $c(X, A)-X$ of $A$ onto $A$ (see our topological definition of the connecting homomorphism $\partial$ given in Equation (4.1)), together with the fact that the odd Chern form on the identity-based loop space of the Fredholm operators transgresses the even Chern form on the Fredholm operators.

Taking a finite CW complex with cohomotopy twist $(X, H)$ now, and replacing $X$ and $A$ in the statement of Theorem 4.18 with skeleta $X^{p}$ and $X^{p-1}$ respectively, we have that the Chern character induces a morphism of spectral sequences from the $\left\{E_{r}(K)\right\}$ of Theorem 4.4 to the $\left\{E_{r}(H)\right\}$ of Theorem 4.15. In particular, on the first page, a choice of trivialisation of the twist over $X^{p}-X^{p-1}$ 
(a disjoint union of contractible spaces) identifies the twisted Chern character with the ordinary Chern character, so that the square

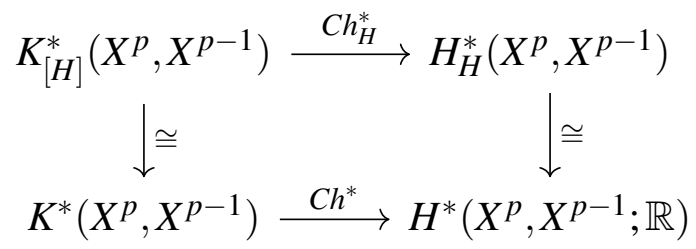

commutes. The expected theorem then follows.

Theorem 4.19. Given any finite $C W$ complex with cohomotopy twist $(X, H)$, the twisted Chern character induces an isomorphism of the real-ised Atiyah-Hirzebruch spectral sequence $\left\{E_{r}(K) \otimes\right.$ $\mathbb{R}\}$ onto the spectral sequence $\left\{E_{r}(H)\right\}$ of Theorem 4.15 computing the higher twisted cohomology. Consequently the Chern character defines a real isomorphism $C_{H}^{*}: K_{[H]}^{*}(X) \otimes \mathbb{R} \rightarrow H_{H}^{*}(X)$.

Remark 4.20. Note that the spectral sequence we have used in Theorem 4.15 to compute higher twisted cohomology distinct from that used by Atiyah and Segal in [4]. Specifically, while our spectral sequence has been constructed from the filtration of the underlying space by its skeleta, that of Atiyah and Segal used a filtration by degree of differential form (we refer the reader to [32] for a detailed construction of the spectral sequence filtered by form degree in the higher twisted case). Our reason for choosing a different spectral sequence is that it is unclear even in the case of the 3-twist considered by Atiyah and Segal why the twisted Chern character induces the claimed morphism of spectral sequences when one filters the twisted de Rham complex by degree instead of by skeleta. Atiyah and Segal claim that this follows "Because the twisted Chern character is functorial" [4, p. 24] - however, as is clear from our presentation, naturality of the Chern character is with respect to $\mathrm{CW}$ pairs and not, a-priori, with respect to degree of differential forms.

In particular, we have not been able to prove that the higher differentials of the real-ised AtiyahHirzebruch spectral sequence are Massey products with the twisting form. This computation appears to require the filtration by degree of differential forms (see [32, Theorem 1.2] for a detailed exposition). Thus to obtain the higher differentials, one must find a way of relating the filtration of higher twisted cohomology by skeleta with the filtration by degree of differential forms. We leave this as an open problem.

\section{REFERENCES}

[1] Arlettaz, D.: The order of the differentials in the Atiyah-Hirzebruch spectral sequence. Ktheory 6 (1992) 3.6, 3.7

[2] Atiyah, M. F: K-theory. Benjamin, New York (1967) 4.1, 4.1

[3] Atiyah M. F., Hirzebruch, F.: Vector bundles and homogeneous spaces. Proc. Sympos. Pure Math. 3, 7-38, (1961) 4.1, 4.1

[4] Atiyah M. F., Segal, G.: Twisted K-theory and cohomology. Inspired by S. S. Chern. Nankai Tracts Math. 11 World Sci. Publ., Hackensack, NJ (2006) (document), 1, 2, 2.1, 2.1, 2.3, 4, 4.1, 4.2, 4.3, 4.3, 4.20

[5] Atiyah M. F., Segal, G.: Twisted K-theory. Ukr. Mat. Visn. 1, 287-330 (2004) (document)

[6] Blackadar, B.: K-theory for operator algebras (Mathematical Sciences Research Institute Publications 5). Springer-Verlag, New York (1986) 1 
[7] Borsuk K.: Sur les groupes des classes de transformations continues. C. R. Acad. Sci. Paris 202, 1400-1403 (1936) 2.3

[8] Borsuk K.: Theory of retracts. Państwowe Wydawn 44. Naukowe (1967) 2.3

[9] Bouwknegt, P., Mathai, V.: D-branes, B-fields and twisted K-theory. J. High Energy Phys. 03, 007, (2000) (document)

[10] Bouwknegt, P., Carey, A., Mathai, V., Murray, M., Stevenson, D.: Twisted K-theory and K-theory of bundle gerbes. Comm. Math. Phys. 228, 17-49 (2002) (document)

[11] Bouwknegt, P., Evslin, J., Mathai, V.: T-duality: Topology Change from H-flux. Comm. Math. Phys. 249, 383-415 (2004)(document), 3.1

[12] _ On the Topology and Flux of T-Dual Manifolds. Phys. Rev. Lett. 92, 181601 (2004) (document), 3.1

[13] Bouwknegt, P., Evslin, J., Mathai, V.: Spherical T-duality. Comm. Math. Phys. 337, 909-954 (2015) (document), 3.1

[14] Bouwknegt, P., Evslin, J., Mathai, V.: Spherical T-duality II: An infinity of spherical T-duals for non-principal $S U$ (2)-bundles. J. Geom. Phys. 92, 46-54 (2015) (document), 3.1, 3.1

[15] Bouwknegt, P., Evslin, J., Mathai, V.: Spherical T-duality and the spherical Fourier-Mukai transform. J. Geom. Phys. 133, 303-314 (2018) 3.1

[16] Brook, D.: Higher Twisted K-theory. MPhil thesis, University of Adelaide (2020) 1

[17] Bunke, U., Schick, T.: On the topology of T-duality. Rev. Math. Phys. 17, 77-112 (2005) (document), 3.1, 3.7

[18] Cavalcanti, G. Gualtieri, M.: Generalized complex geometry and T-duality, in "A Celebration of the Mathematical Legacy of Raoul Bott" (CRM Proceedings \& Lecture Notes), pp. 341366. American Mathematical Society (2010) 3.1

[19] Christensen, J. D., Sinnamon, G., Wu, E.: The D topology for diffeological spaces. Pacific J. Math. 272, 87-110 (2014) 4.2

[20] Cuntz, J.: K-theory for certain $C^{*}$-algebras. Ann. of Math. 113, 181-197 (1981) 1

[21] Dadarlat, M., Pennig, U.: A Dixmier-Douady theory for strongly self-absorbing $C^{*}$-algebras. J. Reine Angew. Math. 718, 153-181 (2016) 1, 2, 2.1, 2.4

[22] Freed, Daniel S., Hopkins, Michael J., Teleman, Constantin: Loop groups and twisted Ktheory I. J. Topol. 4 (2011), no. 4, 737-798. (document)

[23] Gomez Guerra, J.: Models of twisted K-theory. Ph.D. Thesis, University of Michigan (2008) (document)

[24] Griffiths, P., Morgan, J.: Rational Homotopy Theory and Differential Forms, Second Edition (Progress in Mathematics 16), Birkhäuser (2013) 4.2, 4.2

[25] Garmendia, A., Villatoro, J.: Integration of singular foliations via paths. arXiv:1912.02148 [math.DG], (2019) 4.2

[26] Hector, G., Macías-Virgós, E., Sanmartín-Carbón, E.: De Rham cohomology of diffeological spaces and foliations. Indag. Math. 21, 212-220 (2011) 4.2

[27] Hori. K.: D-branes, T-duality, and index theory. Adv. Theor. Math. Phys. 3, 281-342 (1999) (document)

[28] Husemoller, D.: Fibre Bundles. Springer-Verlag, New York (1994) 2.1

[29] Iglesias-Zemmour, P., Karshon, Y., Zadka, M.: Orbifolds as diffeologies. Trans. Am. Math. Soc. 362, 2811-2831 (2010) 4.2

[30] Iglesias-Zemmour, P.: Diffeology (Mathematical Surveys and Monographs 185). American Mathematical Society (2013) 4.2 
[31] Kuribayashi, K.: Simplicial cochain algebras for diffeological spaces. arXiv:1902.10937v5 [math.AT], (2019) 4.2, 4.2

[32] Li, W., Liu, W., Wang, H.: On a Spectral Sequence for Twisted Cohomologies. Chin. Ann. Math. 35B, 633-658 (2014) 4, 4.3, 4.20

[33] Lind, J. A., Sati, H., Westerland, C.: Twisted iterated algebraic K-theory and topological T-duality for sphere bundles. Ann. K-Theory 5, 1-42 (2020) (document)

[34] MacDonald, L. E.: Hierarchies of holonomy groupoids for foliated bundles. arXiv:2004.13929 [math.DG], (2020) 4.2

[35] MacDonald, L. E.: The holonomy groupoids of singularly foliated bundles. arXiv:2006.14271 [math.DG], (2020) 4.2

[36] Madsen, I., Snaith, V., Tornehave, J.: Infinite loop maps in geometric topology. Math. Proc. Cambridge Philos. Soc 81, 399-430 (1977) (document)

[37] Mathai, V., Rosenberg, J.: T-duality for torus bundles with H-fluxes via noncommutative topology. Comm. Math. Phys. 253, 705-721 (2005) (document)

[38] Mathai, V., Stevenson, D.: Chern character in twisted K-theory: equivariant and holomorphic cases. Comm. Math. Phys. 236, 161-186 (2003) (document)

[39] Mathai, V., Wu, S.: Analytic torsion for twisted de Rham complexes. J. Differential Geom. 88, 297-332 (2011) 1

[40] Minasian, R., Moore, G.: K-theory and Ramond-Ramond charge. J. High Energy Phys. (1997). https://doi.org/10.1088/1126-6708/1997/11/002 (document)

[41] Pennig, U.: A noncommutative model for higher twisted K-Theory. J. Topology, 27-50 (2016) (document), 1, 4.1

[42] Polchinski, J.: String theory. Vol. I. An introduction to the bosonic string. Reprint of the 2003 edition (Cambridge Monographs on Mathematical Physics). Cambridge University Press, Cambridge, (2005) (document)

[43] Polchinski, J.: String theory. Vol. II. Superstring theory and beyond. Reprint of 2003 edition (Cambridge Monographs on Mathematical Physics). Cambridge University Press, Cambridge, (2005) (document)

[44] Raeburn, I., Rosenberg, J.: Crossed products of continuous-trace $C^{*}$-algebras by smooth actions. Trans. Amer. Math. Soc. 305, 1-45 (1988) 3.1

[45] Segal, G.: Categories and cohomology theories. Topology 13, 293-312 (1974) (document)

[46] Soriau, J.-M.: Groupes différentiels. In: García P.L., Pérez-Rendón A., Souriau J.M. (eds) Differential Geometrical Methods in Mathematical Physics. Lecture Notes in Mathematics, vol 836. Springer, Berlin, Heidelberg (1980) 4.2

[47] Teleman. C.: K-theory and the moduli space of bundles on a surface and deformations of the Verlinde algebra. In: Topology, geometry and quantum field theory, volume 308 of London Math. Soc. Lecture Note Ser., pp 358-378. Cambridge Univ. Press, Cambridge (2004) (document)

[48] Walschap,G.: The Euler class as a cohomology generator. Illinois J. Math. 46, 165-169 (2002) 3.3

[49] Warner, F. W.: Foundations of Differentiable Manifolds and Lie Groups (Graduate Texts in Mathematics 94). Springer-Verlag, Berlin-Heidelberg (1971) 4.2

[50] Wegge-Olsen, N. E.: $K$-Theory and $C^{*}$-Algebras: A Friendly Approach. Oxford Science Publications, (1993) 1 
[51] C. Westerland, Topological T-duality is twisted Atiyah duality, preprint: arXiv:1503.00210. (document)

[52] Witten. E.: D-branes and K-theory. J. High Energy Phys. (1998). https://doi.org/10.1088/1126-6708/1998/12/019 (document)

School of Mathematical Sciences, University of Adelaide, Adelaide 5005, Australia

Email address: lachlan.macdonald@adelaide.edu.au

School of Mathematical Sciences, University of Adelaide, Adelaide 5005, Australia

Email address: mathai .varghese@adelaide.edu.au

School of Mathematical Sciences, University of Adelaide, Adelaide 5005, Australia

Email address: hemanth. saratchandran@adelaide.edu. au 